\title{
Superrotation charge and supertranslation hair on black holes
}

\author{
Stephen W. Hawking, ${ }^{a}$ Malcolm J. Perry ${ }^{a}$ and Andrew Strominger ${ }^{b}$ \\ ${ }^{a}$ DAMTP, Centre for Mathematical Sciences, University of Cambridge, \\ Wilberforce Road, Cambridge, U.K. \\ ${ }^{b}$ Center for the Fundamental Laws of Nature, Harvard University, \\ 17 Oxford Street, Cambridge, MA, U.S.A. \\ E-mail: swh1@cam.ac.uk, m.j.perry@damtp.cam.ac.uk, \\ strominger@physics.harvard.edu
}

ABSTRACT: It is shown that black hole spacetimes in classical Einstein gravity are characterized by, in addition to their ADM mass $M$, momentum $\vec{P}$, angular momentum $\vec{J}$ and boost charge $\vec{K}$, an infinite head of supertranslation hair. The distinct black holes are distinguished by classical superrotation charges measured at infinity. Solutions with supertranslation hair are diffeomorphic to the Schwarzschild spacetime, but the diffeomorphisms are part of the BMS subgroup and act nontrivially on the physical phase space. It is shown that a black hole can be supertranslated by throwing in an asymmetric shock wave. A leading-order Bondi-gauge expression is derived for the linearized horizon supertranslation charge and shown to generate, via the Dirac bracket, supertranslations on the linearized phase space of gravitational excitations of the horizon. The considerations of this paper are largely classical augmented by comments on their implications for the quantum theory.

KEYwords: Black Holes, Gauge Symmetry, Nonperturbative Effects

ArXiv EPRINT: 1611.09175 


\section{Contents}

1 Introduction 1

2 Supertranslation and superrotation charge conservation 3

2.1 Asymptotic expansion 3

2.2 The scattering problem 6

$\begin{array}{lll}2.3 & \text { Discussion } & 7\end{array}$

3 Asymptotic symmetries $\quad 8$

3.1 Supertranslations 8

$\begin{array}{llr}3.2 & \text { Superrotations } & 9\end{array}$

4 Schwarzschild supertranslations $\quad 10$

5 Implanting supertranslation hair $\quad 11$

6 Classical superrotation charges of supertranslation hair $\quad 13$

7 Horizon charges $\quad \mathbf{1 5}$

$\begin{array}{ll}7.1 \text { Symplectic forms and linearized charges } & 16\end{array}$

$\begin{array}{lll}7.2 & \text { Schwarzschild charges } & 17\end{array}$

$\begin{array}{ll}7.3 \text { Gauge fixing and Dirac brackets } & 19\end{array}$

$\begin{array}{ll}\text { A Some useful formulae } & 24\end{array}$

\section{Introduction}

Over the last few years it has been found [1-14] that empty space is a richer place than was previously believed. ${ }^{1}$ Even the classical Minkowskian vacuum, far from being a unique, featureless configuration, is infinitely degenerate in all electromagnetic, Yang-Mills and gravitational theories. Information about the vacuum configuration is holographically stored at the asymptotic boundary of spacetime. Different vacua are related by infinite-dimensional asymptotic symmetries which, in the quantum theory, can be infinitesimally described as creating or annihilating soft (i.e. zero-energy) particles such as photons or gravitons.

The infinity of associated conserved charges constrain every scattering process in asymptotically Minkowskian spacetimes, including those in which black holes are formed and then evaporate. For each and every conserved charge, the charge on the black hole must be reduced (increased) by exactly the amount carried by any emitted (absorbed) particles [20-23]. Charge conservation is possible only if black holes themselves carry an

\footnotetext{
${ }^{1}$ Prescient early work appears in $[15-19]$.
} 
infinite number of charges or, equivalently, have an infinite head of 'soft hair' [22]. This does not violate the classical no-hair theorems [24] because the distinct black holes are related by diffeomorphisms, albeit 'large' ones which comprise the asymptotic symmetry group and act nontrivially on the classical phase space. Soft hair has implications for the information paradox [25], since charge conservation enforces quantum correlations between the outgoing Hawking quanta and the soft hair configuration.

In this paper we undertake a study the properties of the charges arising from infinitedimensional gravitational symmetries in a weak-coupling expansion. The fundamental definitions of these conserved charges will be given below in terms of simple boundary integrals near spatial infinity. As usual, integration by parts and the constraint equations can be used to express these charges as three-dimensional 'bulk' integrals and thereby associate distinct contributions to the charge from distinct regions of spacetime such as a black hole.

However, even for the simplest of the conserved charges - the ADM energy — this procedure is in the general case fraught with difficulties associated to the choices of slice and gauge. Quantum fluctuations of the spacetime geometry further diminish the utility of such constructions. Nevertheless, in the context of weak coupling, a perturbative analysis of charge conservation in the bulk can be informative. For example it is possible to show, to first order in the gravitational coupling, that the mass of a black hole always increases by the energy flux of radiation across its horizon. A similar picture should exist for all of the conserved charges. For the infinity of electromagnetic charges, such a picture was obtained in [22]. In this paper, while also supplying the reader with some pedagogical background, we continue the program of [22] and perturbatively analyze in some detail the infinity of so-called supertranslation and superrotation symmetries. Supertranslation (superrotation) charge conservation equates the total incoming energy at each angle to the total outgoing energy (angular momentum) at the opposing angle [2, 26].

After spelling out our notation in section 2.1 , in section 2.2 we reiterate the simple origin of the infinity of conserved charges. We show that the very existence of a well-posed scattering problem in asymptotically Minkowskian general relativity requires a boundary condition which matches certain metric components at the top of $\mathcal{I}^{-}$(past null infinity) to those at the bottom of $\mathcal{I}^{+}$(future null infinity). This immediately implies an infinite number of conserved charges, simply from the equality of all the past and future multipole moments of the matched metric data. Explicit expressions are given for the supertranslation charges arising from the matching of the Bondi mass aspect, as well as the superrotation charges arising from the matching of the angular momentum aspect. The relationship to previous work on asymptotic behavior and the peeling theorem is briefly discussed in section 2.3. Section 3.1 reviews the derivation of supertranslation symmetry as the action via Dirac brackets of the supertranslation charges on the physical phase space. Section 3.2 reviews the current status of efforts to similarly associate a symmetry superrotation charge conservation. In section 4 we use the Bondi gauge to continue supertranslations from the boundary into the bulk of the Schwarzschild geometry. The Bondi-gauge metric of an infinitesimally supertranslated Schwarzschild black hole, i.e. a black hole with supertranslation hair, is derived. In section 5 we show, via an explicit Vaidya-type solution, how a black hole can be physically supertranslated by throwing in an asymmetric null shock 
wave. Supertranslated black holes do not carry supertranslation charge because the group is abelian. However in section 6 we find they can and do carry superrotation charges and an explicit expression is given. This provides a classical diagnostic of supertranslation hair (see also $[27,28]$ ). Section 7 gives a canonical construction of the generators of linearized Bondi-gauge supertranslations on the future Schwarzschild horizon $\mathcal{H}^{+}$. Section 7.1 reviews the covariant canonical formalism and symplectic form in gravity. Section 7.2 presents the covariant supertranslation charge $\hat{Q}_{f}^{\mathcal{H}^{+}}$on the horizon. In section 7.3, a careful fixing of the residual gauge symmetries in Bondi gauge which (unlike supertranslations) are zero eigenvalues of the pre-symplectic form is performed. The symplectic form is then inverted on the physical phase space to obtain the Dirac bracket. Finally it is shown that the charge $\hat{Q}_{f}^{\mathcal{H}^{+}}$properly generates horizon supertranslations.

During the course of this work strongly overlapping results were independently obtained in $[27,28]$. Related work has also appeared in [29-74]. We expect our horizon analysis is closely related to much earlier work [75-78] employing different gauges and formalisms. Soft hair appears to be an alternate description of the phenomenon of edge modes as discussed in [79-83]. A precise characterization of the relation of these edge modes and soft hair would be of great interest.

We set Newton's constant $G=1$ throughout.

\section{Supertranslation and superrotation charge conservation}

In this section we review a few salient facts about asymptotically flat spacetimes in classical general relativity (GR) and the newly-discovered infinite number of conserved supertranslation [2] and superrotation [7, 26, 84] charges. Moreover, we show that the existence of this infinite number of conserved charges in GR follows simply from the requirement of a well-posed scattering problem.

\subsection{Asymptotic expansion}

Near future null infinity $\left(\mathcal{I}^{+}\right)$we use retarded coordinates $\left(u, r, \Theta^{A}\right)$ and the Bondi gauge, in which

$$
g_{r A}=g_{r r}=0, \quad \operatorname{det}\left(\frac{g_{A B}}{r^{2}}\right)=g(\Theta) .
$$

Surfaces of constant retarded time $u=t-r$ are null. $\mathcal{I}^{+}$is the surface $r \rightarrow \infty$. $\Theta^{A}$ are coordinates on the two-sphere and $g(\Theta)$ is a fixed function on the sphere. An asymptotically flat metric has a large $r$-expansion ${ }^{2}$

$$
\begin{aligned}
d s^{2}= & -d u^{2}-2 d u d r+r^{2} \gamma_{A B} d \Theta^{A} d \Theta^{B} \\
& +\frac{2 m}{r} d u^{2}+r C_{A B} d \Theta^{A} d \Theta^{B}+D^{B} C_{A B} d u d \Theta^{A} \\
& +\frac{1}{16 r^{2}} C_{A B} C^{A B} d u d r
\end{aligned}
$$

\footnotetext{
${ }^{2}$ See [85-88] and [89] for recent reviews. We omit here interesting logarithmic terms [90, 91] of potential relevance in the present context.
} 


$$
\begin{aligned}
& +\frac{1}{r}\left(\frac{4}{3} N_{A}+\frac{4 u}{3} \partial_{A} m-\frac{1}{8} \partial_{A}\left(C_{B D} C^{B D}\right)\right) d u d \Theta^{A} \\
& +\frac{1}{4} \gamma_{A B} C_{C D} C^{C D} d \Theta^{A} d \Theta^{B} \\
& +\ldots \ldots
\end{aligned}
$$

where indices are raised and lowered using the metric on the unit sphere, $\gamma_{A B}$. The traceless tensor $C_{A B}$, the Bondi mass aspect $m$, and the angular momentum aspect $N_{A}$ all depend on the $\mathcal{I}^{+}$coordinates $\left(u, \Theta^{A}\right)$ but not $r$. Our definition of $N_{A}^{3}$ differs from the conventional ones in two ways. Firstly, by a shift of $u \partial_{A} m$ which has the advantage that, as can be seen from the constraint equations below, is typically finite for $u \rightarrow \pm \infty$. Secondly, there is a shift of $N_{A}$ by quadratic terms in $C_{A B}$ to obtain a simple relation to the Riemann tensor

$$
\lim _{r \rightarrow \infty} r^{3} R_{A r r u}=N_{A}+u \partial_{A} m
$$

The Bondi news

$$
N_{A B}=\partial_{u} C_{A B}
$$

characterizes the gravitational radiation passing through $\mathcal{I}^{+}$.

The Cauchy data on $\mathcal{I}^{+}$for the full spacetime metric includes ${ }^{4} C_{A B}, m$ and $N_{A}$ which are subject to the constraint equations

$$
\begin{aligned}
\partial_{u} m & =\frac{1}{4} D^{A} D^{B} N_{A B}-T_{u u}, \\
T_{u u} & \equiv \frac{1}{8} N_{A B} N^{A B}+4 \pi \lim _{r \rightarrow \infty}\left[r^{2} T_{u u}^{M}\right], \\
\partial_{u} N_{A} & =-\frac{1}{4} D^{B}\left(D_{B} D^{C} C_{C A}-D_{A} D^{C} C_{B C}\right)+u \partial_{A}\left(T_{u u}-\frac{1}{4} D^{B} D^{C} N_{B C}\right)-T_{u A}, \\
T_{u A} & \equiv 8 \pi \lim _{r \rightarrow \infty}\left[r^{2} T_{u A}^{M}\right]-\frac{1}{4} \partial_{A}\left(C_{B D} N^{B D}\right)+\frac{1}{4} D_{B}\left(C^{B C} N_{C A}\right)-\frac{1}{2} C_{A B} D_{C} N^{B C} .
\end{aligned}
$$

Here $T_{a b}^{M}$ is the matter stress tensor while $T_{a b}$ incorporates corrections from the stress tensor for linearized gravity waves.

The traceless Bondi news $N_{A B}(u, \Theta)$ comprises two unconstrained real functions on $\mathcal{I}^{+}$as expected for the two helicities of the massless graviton. We assume that near the past and future boundaries of $\mathcal{I}^{+}, \mathcal{I}_{+}^{+}$and $\mathcal{I}_{-}^{+}$, the news falls off faster than $\frac{1}{|u|}$ and that the angular momentum aspect $N_{A}$ approaches a finite one-form on $S^{2}$. These (and stronger) asymptotic boundary conditions were proven by Christodoulou and Klainerman [92] to hold in a finite neighborhood of flat space: here we shall consider spacetimes with this asymptotic behavior but do not require them to be near flat space in the deep interior. The news then trivially determines $C_{A B}$ up to an integration function by integrating (2.4).

\footnotetext{
${ }^{3}$ Our angular momentum aspect $N_{A}$ can be related to that defined by Barnich and Troessaert [85-88], $N_{A}^{B T}$ by the following $N_{A}=N_{A}^{B T}-D_{A} m+\frac{1}{4} C_{A B} D_{C} C^{B C}+\frac{3}{16} C^{B C} D_{A} C_{B C}$.

${ }^{4}$ Determining if or when these (or the $\mathcal{I}^{-}$counterparts) comprise a complete set of Cauchy data is an outstanding problem in mathematical relativity which we do not address here. Among other issues are the possibilities of logarithms and further integration functions appearing at higher order in the $\frac{1}{r}$ expansion. See for example [90, 91].
} 
We take the integration function to be $\left.C_{A B}\right|_{\mathcal{I}_{-}^{+}}$. Finiteness of $\left.N_{A}\right|_{\mathcal{I}_{-}^{+}}$and (2.6) then imply $\left.C_{A B}\right|_{\mathcal{I}_{-}^{+}}$is determined from a single real function $C$ on $\mathcal{I}_{-}^{+}[2]$ :

$$
\left.C_{A B}\right|_{\mathcal{I}_{-}^{+}}=-\left.2 D_{A} D_{B} C\right|_{\mathcal{I}_{-}^{+}}+\left.\gamma_{A B} D^{2} C\right|_{\mathcal{I}_{-}^{+}}
$$

Given the news tensor and this initial data at $\mathcal{I}_{-}^{+}$, the constraints may be integrated to give the mass and angular momentum aspects $m$ and $N_{A}$ everywhere on $\mathcal{I}^{+}$. Hence the Cauchy data includes

$$
\left\{N_{A B}(u, \Theta),\left.C(\Theta)\right|_{\mathcal{I}_{-}^{+}},\left.m(\Theta)\right|_{\mathcal{I}_{-}^{+}},\left.N_{A}(\Theta)\right|_{\mathcal{I}_{-}^{+}}\right\}
$$

The Cauchy data (2.8) transforms non-trivially under the BMS+ $\operatorname{subgroup}^{5}[93,94]$ of diffeomorphisms acting near $\mathcal{I}^{+}$, which includes boosts, rotations and supertranslations (formulae for which are in the next section). As shown in [93, 94], despite being diffeomorphic, data sets which differ by BMS transformations are physically inequivalent. For example they can change the ADM energy or transform a configuration with gravity waves simultaneously emerging at the north and south pole on $\mathcal{I}^{+}$into ones where they appear with an arbitrary relative retarded time delay. Even when the news is zero, BMS+ generically changes the vacuum to an inequivalent one with different values of both $\left.C\right|_{\mathcal{I}_{-}^{+}}$and ADM angular momentum. That is, there is an infinite family of inequivalent vacua in GR.

A similar set of equations apply near $\mathcal{I}^{-}$, where we employ advanced Bondi coordinates $\left(v, r, \Theta^{A}\right)$ in which the metric has the asymptotic expansion

$$
\begin{aligned}
d s^{2}= & -d v^{2}+2 d v d r+r^{2} \gamma_{A B} d \Theta^{A} d \Theta^{B} \\
& +\frac{2 m}{r} d v^{2}+r C_{A B} d \Theta^{A} d \Theta^{B}-D^{B} C_{A B} d v d \Theta^{A} \\
& -\frac{1}{16 r^{2}} C_{A B} C^{A B} d v d r \\
& -\frac{1}{r}\left(\frac{4}{3} N_{A}-\frac{4 v}{3} \partial_{A} m-\frac{1}{8} \partial_{A}\left(C_{B D} C^{B D}\right)\right) d v d \Theta^{A} \\
& +\frac{1}{4} \gamma_{A B} C_{C D} C^{C D} d \Theta^{A} d \Theta^{B} \\
& +\ldots \ldots
\end{aligned}
$$

In flat Minkowski space advanced and retarded Bondi coordinates are related by

$$
\left(v, r, \Theta^{A}\right)=\left(u+2 r, r, P \Theta^{A}\right)
$$

where $P \Theta^{A}$ is the antipode of $\Theta^{A}$ on the sphere. ${ }^{6}$ The analog of the Cauchy data (2.8) for $\mathcal{I}^{-}$is

$$
\left\{N_{A B}(v, \Theta),\left.C(\Theta)\right|_{\mathcal{I}_{+}^{-}},\left.m(\Theta)\right|_{\mathcal{I}_{+}^{-}},\left.N_{A}(\Theta)\right|_{\mathcal{I}_{+}^{-}}\right\} .
$$

\footnotetext{
${ }^{5}$ The ' + ' signifies the action is on $\mathcal{I}^{+}$rather than $\mathcal{I}^{-}$.

${ }^{6}$ For standard angular coordinates $\Theta^{A} \sim(\theta, \phi), \quad P \Theta^{A} \sim(\pi-\theta, \phi+\pi)$. This coordinate convention is chosen to simplify the past-future matching conditions below.
} 


\subsection{The scattering problem}

The scattering problem in classical general relativity is, roughly speaking, to find the map from Cauchy data on $\mathcal{I}^{-}$to that on $\mathcal{I}^{+} .{ }^{7}$ Such a map is not even formally determined from the maximal Cauchy development of the $\mathcal{I}^{-}$data (2.11) with the Einstein equation. This determines the data on $\mathcal{I}^{+}$at most up to a BMS+ transformation. A prescription is needed to attach $\mathcal{I}^{+}$, choose a BMS+ frame and determine the initial values for integrating $m$ and $N_{A}$ along $\mathcal{I}^{+}$using the constraints. Without such a prescription, the scattering problem in GR is not defined. In [2], it was proposed that the BMS+ frame should be determined by the Lorentz and CPT invariant matching conditions

$$
\left.C\right|_{\mathcal{I}_{-}^{+}}(\Theta)=\left.C(\Theta)\right|_{\mathcal{I}_{+}^{-}},\left.\quad m(\Theta)\right|_{\mathcal{I}_{-}^{+}}=\left.m(\Theta)\right|_{\mathcal{I}_{+}^{-}},
$$

and in [26] the matching condition for the angular momentum aspect

$$
\left.N_{A}(\Theta)\right|_{\mathcal{I}_{-}^{+}}=\left.N_{A}(\Theta)\right|_{\mathcal{I}_{+}^{-}}
$$

was proposed. This breaks the combined BMS $+\otimes \mathrm{BMS}-$ action on $\mathcal{I}^{+}$and $\mathcal{I}^{-}$down to the diagonal subgroup which preserves these conditions. Noting our convention (2.10) relating $\Theta^{A}\left(\mathcal{I}_{-}^{+}\right)$and $\Theta^{A}\left(\mathcal{I}_{+}^{-}\right)$, we see that (2.12) and (2.13) antipodally equate past and future fields near spatial infinity. At first sight, this antipodal relation appears rather bizarre. However, we expect that it is the only Lorentz and CPT invariant choice and is implicit in most or all GR computations in asymptotically flat spacetimes. In [3] the matching condition (2.12) was in fact proven to be implicit to all orders in standard weak field perturbation theory by demonstrating its equivalence to Weinberg's soft graviton theorem [95]. In [84, 96, 97] a new subleading soft graviton theorem was proven to all orders using Feynman tree diagrams, ${ }^{8}$ and then shown to imply $(2.13)[7,26]$. Motivated by this perturbative analysis, we propose that $(2.12),(2.13)$ are part of the definition of the scattering problem whenever the fields are sufficiently weak near spatial infinity, even if the interior contains a black hole.

The matching conditions (2.12) and (2.13) immediately imply that an infinite number of charges are conserved in GR scattering. Two families of charges are defined at $\mathcal{I}_{-}^{+}$and $\mathcal{I}_{+}^{-}$by:

$$
Q_{f}^{+}=\frac{1}{4 \pi} \int_{\mathcal{I}_{-}^{+}} d^{2} \Theta \sqrt{\gamma} f m, \quad Q_{f}^{-}=\frac{1}{4 \pi} \int_{\mathcal{I}_{+}^{-}} d^{2} \Theta \sqrt{\gamma} f m,
$$

where $f(\Theta)$ is any function on $S^{2}$. Integrating by parts and using the constraint (2.5), these can be written as integrals over $\mathcal{I}^{+}$or $\mathcal{I}^{-}$respectively: ${ }^{9}$

$$
\begin{aligned}
Q_{f}^{+} & =\frac{1}{4 \pi} \int_{\mathcal{I}^{+}} d u d^{2} \Theta \sqrt{\gamma} f\left(T_{u u}-\frac{1}{4} D^{A} D^{B} N_{A B}\right), \\
Q_{f}^{-} & =\frac{1}{4 \pi} \int_{\mathcal{I}^{-}} d v d^{2} \Theta \sqrt{\gamma} f\left(T_{v v}-\frac{1}{4} D^{A} D^{B} N_{A B}\right) .
\end{aligned}
$$

\footnotetext{
${ }^{7}$ Of course if a black hole is formed we need Cauchy data on $\mathcal{I}^{+} \cup \mathcal{H}^{+}$, where $\mathcal{H}^{+}$is the future horizon, but this section is mainly concerned with the weak-field problem for which black holes are absent.

${ }^{8}$ Although this paper largely concerns classical GR, we note that (2.13) is possibly deformed by an anomaly at one loop [98-103]. Since some matching relation of the form (2.13) must exist in order for gravitational scattering to be defined, this suggests that these one loop corrections deform rather than eliminate the conserved charges. This is an important open problem. Some recent progress has appeared in [104, 105].

${ }^{9}$ In the presence of massive matter or black holes there are extra contibutions at $\mathcal{I}_{+}^{+}$and $\mathcal{I}_{-}^{-}$.
} 
(2.12) implies:

$$
Q_{f}^{+}=Q_{f}^{-} .
$$

The case $f=1$ is just the total energy conservation while the $\ell=1$ harmonic $f=Y_{m}^{1}$ gives the well known ADM momentum conservation. The general case (2.16) provides an infinite number of new generalizations of these four laws referred to as supertranslation charge conservation [2]. Choosing $f$ to be a delta function, the generalized conservation law equates the net incoming energy flux at each angle (including linear gravitational terms) to the net outgoing energy flux at the opposing angle. The relation of these charges to supertranslation symmetry will be discussed in the next section.

A second infinity of conserved charges can similarly be constructed from an arbitrary vector field $Y^{A}$ on the sphere. Using (2.13) one finds

$$
Q_{Y}^{+}=\frac{1}{8 \pi} \int_{\mathcal{I}_{-}^{+}} d^{2} \Theta \sqrt{\gamma} Y^{A} N_{A}=\frac{1}{8 \pi} \int_{\mathcal{I}_{+}^{-}} d^{2} \Theta \sqrt{\gamma} Y^{A} N_{A}=Q_{Y}^{-}
$$

This expresses conservation of superrotation charge. The special cases for which $Y^{A}$ is one of the 6 global conformal Killing vectors on $S^{2}$ are conservation of ADM angular momentum and boost charge, sometimes referred to as the BORT (Beig-O'Murchada-ReggeTeitelboim) [106, 107] center-of-mass. Choosing the vector field to be a delta-function, these new conservation laws equate net in and out angular momentum flux for every angle.

The supertranslation and superrotation charges are absolutely conserved in the sense that each gives a number constructed according to (2.15) from incoming classical data on $\mathcal{I}^{-}$that must equal a number constructed from outgoing data on $\mathcal{I}^{+}$. This same number can also be constructed from data on any spacelike slice that ends on $\mathcal{I}_{-}^{+}$or $\mathcal{I}_{+}^{-}$. This is qualitatively different from e.g. the oft-discussed Bondi mass as a function of retarded time which is not conserved but rather obeys a conservation law relating its time dependence to energy flux through $\mathcal{I}^{+}$.

The existence of these conserved charges is in principle experimentally verifiable. Indeed, proposed tests of the gravitational memory effect, although not initially recognized as such, are tests of supertranslation charge conservation [20]. Superrotation charge conservation may in principle be tested via the gravitational spin memory effect [21].

In conclusion, the very existence of a well-posed scattering problem from $\mathcal{I}^{-}$to $\mathcal{I}^{+}$in GR necessitates the existence, for any matching condition, of an infinite number of conserved supertranslation and superrotation charges. With our matching conditions (2.12), (2.13) the explicit expressions for these charges are in (2.14), (2.17).

\subsection{Discussion}

It may seem peculiar that this infinity of exactly conserved charges, which generalize ADM energy and angular momentum, has gone unnoticed in the more than half a century since the notion of an asymptotically flat spacetime was introduced in [108]. Part of the reason for this is that many early studies concentrated on special spacetimes in which the peeling theorem [109] applies and Penrose's conformal compactification [110] can be utilized. In fact the peeling theorem does not apply in generic physical settings, see e.g. [111, 112] . A 
simple example which violates peeling is a pair of massive bodies coming in from infinity with asymptotically constant velocities and no incoming radiation. In this type of situation, however, peeling can typically be restored by adding incoming radiation in just such a finetuned way that the solution is exactly Schwarzschild outside some arbitrarily large but finite radius [113-115]. This procedure fine-tunes all of the nontrivial supertranslation and superrotation charges to zero, rendering the conservation laws rather trivial. It was a commonly held expectation that, in the generic physical case, the singularity structure near spatial infinity is too uncontrolled to admit well-defined conserved charges of the type described here. If correct, this would suggest that there are no physical contexts in which an infinite number of non-trivial and well-defined conserved charges can exist. This all changed relatively recently starting from the work of Christodoulou and Klainerman [92], who showed ${ }^{10}$ that generic spacetimes in a finite neighborhood of flat space lie precisely in the sweet spot where it is possible to define $[2,7,26]$ an infinite number of finite, generically non-zero and conserved supertranslation and superrotation charges. In this paper we consider a larger family of spacetimes whose asymptotics lie in the same sweet spot and have the conserved charges, but are not necessarily in a small neighborhood of flat space and may contain black holes in the interior.

\section{$3 \quad$ Asymptotic symmetries}

It is typically the case that conserved charges imply symmetries. In judicious circumstances, a physical phase space $\Gamma$ can be constructed by imposing suitable constraints and gauge conditions. Dirac's procedure is then applied to give the Dirac bracket $\{$,$\} . One then$ defines the infinitesimal symmetry associated to a conserved charge $Q$ on the fields $\Phi$ by

$$
\delta \Phi=\{Q, \Phi\} .
$$

In practice, many subtleties may arise in implementing this procedure including the identification of proper boundary conditions and zero modes. As reviewed in this section, the program has been completed for supertranslations but remains underway for superrotations.

\subsection{Supertranslations}

Dirac brackets involving $C_{A B}$ (including its zero modes) were constructed in [3]. ${ }^{11}$ Commutators of the supertranslation charge $Q_{f}^{+}$in (2.14) were then shown to obey

$$
\begin{aligned}
\left\{Q_{f}^{+}, C_{A B}\right\} & =f \partial_{u} C_{A B}-2 D_{A} D_{B} f+\gamma_{A B} D^{2} f, \\
\left\{Q_{f}^{+},\left.C\right|_{\mathcal{I}_{-}^{+}}\right\} & =f .
\end{aligned}
$$

\footnotetext{
${ }^{10}$ The key result of [92] relevant for our purposes is that the Bondi news falls off at least as fast as $\frac{1}{|u|^{3 / 2}}$ (or $\frac{1}{|v|^{3 / 2}}$ ) near the boundaries of $\mathcal{I}$. This is much faster than required for finiteness of the total radiated energy, and in particular implies that $C_{A B}$ is finite and well defined at the boundaries of $\mathcal{I}$, enabling the fundamental identification (2.12). If the news decayed only as $\frac{1}{|u|}, C_{A B}$ would diverge and the scattering problem would be ill-posed.

${ }^{11}$ This refined the results of $[15-18,116,118,119]$ by a careful treatment of zero modes, including an imposition as physical constraints of the vanishing of the Weyl tensor and the Bondi news at the boundaries of $\mathcal{I}$.
} 
This is easily recognized as the action on $C_{A B}$ of the BMS+ supertranslations $[93,94]$ which are diffeomorphisms generated by the vector field

$$
\zeta_{f}=f \partial_{u}-\frac{1}{r} D^{A} f \partial_{A}+\frac{1}{2} D^{2} f \partial_{r}+\ldots
$$

Here the subleading $\frac{1}{r}$ corrections required to preserve Bondi gauge depend on the metric and $D^{2} \equiv \gamma^{A B} D_{A} D_{B}$. The full BMS+ group is a semidirect product of supertranslations with the Lorentz group.

The fact that the symmetry generated by $Q_{f}$ is a subgroup of a known symmetry (diffeomorphisms) of the standard presentation of the theory is a beautiful feature of this example. It is not obvious or a priori guaranteed. Indeed there are a number of examples (e.g. [31]) where this is not the case.

Interestingly, the vacuum solution $C_{A B}=0$ on $\mathcal{I}^{+}$is not invariant under supertranslations. In other words, supertranslation symmetry is spontaneously broken. There are an infinite number of degenerate classical vacua labelled by the function $\left.C\right|_{\mathcal{I}^{+}}$, each of which is preserved by a different Poincare subgroup of BMS+. These vacua have different ADM angular momenta. This is consistent with the existence of vacuum solutions with nonzero angular momentum [120]. This is sometimes referred to as the 'problem of angular momentum' in GR. However properly understood it is a beautiful feature indicating a rich vacuum structure, not a problem!

\subsection{Superrotations}

It is natural to expect that superrotation charges canonically generate the antipodallyidentified Virasoro-like symmetry presented in [26, 85-88] whose global $\operatorname{SL}(2, C)$ subgroup is the Lorentz group. We think this is likely in some sense the case. However superrotation symmetry is more subtle than its supertranslation analog and the construction has not been completed. The difficulty can be seen in a naive application of the Dirac brackets of [3] which yield

$$
\left\{Q_{Y}^{+}, N_{A B}\right\}=\mathcal{L}_{Y} N_{A B}-D_{A} D_{B} D_{C} Y^{C}+\frac{1}{2} \gamma_{A B} D^{2} D_{C} Y^{C}
$$

Apparently $Q_{Y}^{+}$does not preserve the condition that $N_{A B}$ vanish at the boundaries of $\mathcal{I}^{+}$: i.e. it does not map the phase space considered in [3] into itself. Quantum mechanically, the action of $Q_{Y}^{+}$will produce a state outside the Hilbert space studied in [3]. A larger phase space and associated bracket is needed, but has not yet been found. Indeed recent work [121] building on [122] has shown have shown that superrotations can create strings which pierce $\mathcal{I}$ and destroy asymptotic flatness, suggesting the requisite phase space is the one considered in [123]. Other very interesting recent works have suggested that superrotations can be understood in terms of diffeomorphisms which violate standard falloff conditions $[45,121,124,125]$. An important issue for the quantum theory is the appearance of one loop corrections [98] which depend on the order of soft limits [99]. These and other important issues are beyond the scope of this paper (although in section 7.3 
we will show that non-holomorphic superrotations preserve Bondi gauge). Early discussions of superrotation symmetry can be found in [122, 126, 127], and more recent ones in $[7,26,27,84-88,104,105,128]$.

In this paper we will not use the superrotation symmetry per se - only the finite and conserved superrotation charge given by (2.17), and defer the above interesting issues to future work.

\section{Schwarzschild supertranslations}

In this section we will describe the infinitesimal supertranslation of the Schwarzschild black hole i.e. a black hole with linearized supertranslation hair. This specializes more general formulae which can be found in [85-88]. This type of soft hair appears to be an alternate description of the edge modes as discussed in [79-83].

The extension of an asymptotic gauge symmetry into the interior is gauge dependent. In a general time dependent situation, there is unlikely to be a useful or canonical choice of gauge. Quantum fluctuations further diminish the utility of specific choices. In quantum gravity in asymptotic Minkowski space, we expect the only fully well-defined observables are supported at the boundary at infinity.

It is nevertheless sometimes possible, armed with a gauge choice, to define interior quantities such as local gravitational energy densities at first non-trivial order in perturbation theory around Schwarzschild. This is sometimes useful in developing a picture and intuition for the behavior of the spacetime away from its boundary. For example one may show at leading order in perturbation theory that, at both the classical and quantum level, the total energy comprised of linearized perturbations plus the mass of the black hole itself is conserved. Moreover, this perturbative conservation law is the linearization of an exact, nonperturbative conservation law, which can only be exactly phrased in terms of asymptotic quantities. It is in this spirit that we study the linearized action of supertranslations in Schwarzschild.

In advanced Bondi coordinates the Schwarzschild metric is

$$
d s^{2}=-V d v^{2}+2 d v d r+r^{2} \gamma_{A B} d \Theta^{A} d \Theta^{B}, \quad V \equiv 1-\frac{2 M}{r} .
$$

We wish to find the BMS - supertranslations $\zeta$ which preserve Bondi gauge (2.1) and the standard metric component falloffs at large $r$ while having bounded components in a local orthonormal frame at large $r$. The last condition eliminates all superrotations, including boosts and rotations. The former conditions require, for Schwarzschild

$$
\begin{aligned}
\mathcal{L}_{\zeta} g_{r A} & =\partial_{A} \zeta^{v}+g_{A B} \partial_{r} \zeta^{B}=0, \\
\mathcal{L}_{\zeta} g_{r r} & =2 \partial_{r} \zeta^{v}=0, \\
\frac{r}{2} g^{A B} \mathcal{L}_{\zeta} g_{A B} & =r D_{A} \zeta^{A}+2 \zeta^{r}=0 .
\end{aligned}
$$

The general solution to this consistent with Bondi gauge and asymptotic falloffs is ${ }^{12}$

$$
\zeta_{f}=f \partial_{v}+\frac{1}{r} D^{A} f \partial_{A}-\frac{1}{2} D^{2} f \partial_{r}, \quad \partial_{r} f=\partial_{v} f=0 .
$$

\footnotetext{
${ }^{12}$ The general solution without restricted falloffs is given in section 7 below.
} 
This extends the asymptotic expansion of the supertranslations on $\mathcal{I}^{-}$to the entire region covered by the advanced coordinates. This includes $\mathcal{I}^{-}$and $\mathcal{H}^{+}$but not $\mathcal{I}^{+}$. These act on the Schwarzschild metric as

$$
\begin{aligned}
\mathcal{L}_{f} g_{v v} & =\frac{M D^{2} f}{r^{2}} \\
\mathcal{L}_{f} g_{A B} & =2 r D_{A} D_{B} f-r \gamma_{A B} D^{2} f \\
\mathcal{L}_{f} g_{A v} & =-D_{A}\left(V f+\frac{1}{2} D^{2} f\right) .
\end{aligned}
$$

Adding this to (4.1) gives the infinitesimally supertranslated Schwarzschild geometry: ${ }^{13}$

$$
\begin{aligned}
d s^{2}= & -\left(V-\frac{M D^{2} f}{r^{2}}\right) d v^{2}+2 d v d r-d v d \Theta^{A} D_{A}\left(2 V f+D^{2} f\right) \\
& +\left(r^{2} \gamma_{A B}+2 r D_{A} D_{B} f-r \gamma_{A B} D^{2} f\right) d \Theta^{A} d \Theta^{B} .
\end{aligned}
$$

The event horizon is at $r=2 M+\frac{1}{2} D^{2} f$. This describes a black hole with linearized supertranslation hair.

\section{Implanting supertranslation hair}

In the previous sections we described a supertranslated eternal Schwarzschild black hole. In order to be certain such objects really exist, in this section we describe how one physically makes such a hairy black hole.

First we show how to add supertranslation hair to bald eternal Schwarzschild, and then generalize to a black hole formed from the vacuum. At advanced time $v_{0}$ in Schwarzschild we send in a linearized shock wave with energy momentum density

$$
\hat{T}_{v v}=\frac{\mu+\hat{T}(\Theta)}{4 \pi r^{2}} \delta\left(v-v_{0}\right)
$$

near $\mathcal{I}^{-}$. We wish to solve for the linearized metric in such a way that the solution is diffeomorphic to Schwarzschild both before and after the shock wave. Stress energy conservation $\nabla_{a} \hat{T}^{a b}=0$ then mandates subleading in $\frac{1}{r}$ corrections to the stress tensor for shock waves which are not spherically symmetric. These take the form ${ }^{14}$

$$
\hat{T}_{v v}=\left(\frac{\mu+\hat{T}}{4 \pi r^{2}}+\frac{\hat{T}^{(1)}}{4 \pi r^{3}}\right) \delta\left(v-v_{0}\right), \quad \hat{T}_{v A}=\frac{\hat{T}_{A}}{4 \pi r^{2}} \delta\left(v-v_{0}\right) .
$$

where $\hat{T}(\Theta)$ has only $\ell>1$ components $^{15}$ so that and $\hat{T}^{(1)}$ and $\hat{T}_{A}$ are functions of $x$ determined by

$$
\left(D^{2}+2\right) \hat{T}^{(1)}=-6 M \hat{T}, \quad D^{A} \hat{T}_{A}=\hat{T}^{(1)} .
$$

\footnotetext{
${ }^{13}$ It may be possible to find the finitely supertranslated geometry. This was accomplished at null infinity in [34] and related finite problems were solved in [28, 122, 129-131]. However in this paper our attention is restricted to the linearized theory.

${ }^{14}$ We are grateful to Alex Lupsasca, Monica Pate and Prahar Mitra for help with this solution.

${ }^{15}$ The $\ell=0$ component is represented by $\mu$. The $\ell=1$ component, which would add $A D M$ momentum to the black hole, is eliminated to simplify the discussion.
} 
The solutions are conveniently expressed by introducing the Green function solving

$$
\frac{\sqrt{\gamma}}{4} D^{2}\left(D^{2}+2\right) G\left(\Theta ; \Theta^{\prime}\right)=\delta^{2}(\Delta \Theta)
$$

namely [20]

$$
G\left(\Theta ; \Theta^{\prime}\right)=\frac{1}{\pi} \sin ^{2} \frac{\Delta \Theta}{2} \log \sin ^{2} \frac{\Delta \Theta}{2},
$$

where $\Delta \Theta$ is the angle on the sphere between $\Theta$ and $\Theta^{\prime}$. Further defining

$$
\hat{C}(\Theta) \equiv \int d^{2} \Theta^{\prime} G\left(\Theta, \Theta^{\prime}\right) \hat{T}\left(\Theta^{\prime}\right)
$$

(5.3) has the solution

$$
\begin{aligned}
\hat{T} & =\frac{1}{4} D^{2}\left(D^{2}+2\right) \hat{C}, \\
\hat{T}^{(1)} & =-\frac{3 M}{2} D^{2} \hat{C}, \\
\hat{T}_{A} & =-\frac{3 M}{2} \partial_{A} \hat{C} .
\end{aligned}
$$

Equivalently

$$
\begin{aligned}
& \hat{T}_{v v}=\frac{1}{4 \pi r^{2}}\left[\mu+\frac{1}{4} D^{2}\left(D^{2}+2\right) \hat{C}-\frac{3 M}{2 r} D^{2} \hat{C}\right] \delta\left(v-v_{0}\right), \\
& \hat{T}_{v A}=-\frac{3 M}{8 \pi r^{2}} D_{A} \hat{C} \delta\left(v-v_{0}\right) .
\end{aligned}
$$

The leading large- $r$ constraint equation on $\mathcal{I}^{-}$may then be written

$$
\partial_{v} m=\frac{1}{4} D^{A} D^{B} N_{A B}+(\mu+\hat{T}(\Theta)) \delta\left(v-v_{0}\right) .
$$

This equation constrains, but does not fully determine, the mass aspect $m$ and $C_{A B}$. We wish to solve it in such a way that $\partial_{A} m=0$ everywhere. Integrating over the sphere this implies

$$
m=M+\mu \theta\left(v-v_{0}\right)
$$

It then follows from (2.5) that

$$
D_{A} D_{B} C^{A B}=-4 \hat{T}(\Theta) \theta\left(v-v_{0}\right) .
$$

The unique solution to this is

$$
C_{A B}=-2 \theta\left(v-v_{0}\right)\left(D_{A} D_{B} \hat{C}-\frac{1}{2} \gamma_{A B} D^{2} \hat{C}\right) .
$$

One may verify that

$$
\begin{aligned}
h_{v v} & =\theta\left(v-v_{0}\right)\left(\frac{2 \mu}{r}-\frac{M D^{2} \hat{C}}{r^{2}}\right), \\
h_{A B} & =-2 r \theta\left(v-v_{0}\right)\left(D_{A} D_{B} \hat{C}-\frac{1}{2} \gamma_{A B} D^{2} \hat{C}\right), \\
h_{v A} & =\theta\left(v-v_{0}\right) \partial_{A}\left(1-\frac{2 M}{r}+\frac{1}{2} D^{2}\right) \hat{C},
\end{aligned}
$$


solves the linearized Einstein equation with source (5.8) for all $r$ and hence are the complete linearized metric perturbations. Comparing with the formula (4.6) for a supertranslation of Schwarzschild one finds (5.13) can be written

$$
h_{a b}=\theta\left(v-v_{0}\right)\left(\mathcal{L}_{f=-\hat{C}} g_{a b}+\frac{2 \mu}{r} \delta_{a}^{v} \delta_{b}^{v}\right)
$$

Hence the shock wave is a domain wall interpolating between two BMS inequivalent Schwarzschild vacua, whose mass parameters differ by $\mu$.

The shock wave induces a shift in the transverse components of the metric perturbation on the horizon. Integrating over a null generator of the horizon

$$
\Delta h_{A B}(r=2 M, v, \Theta)=\int d v \partial_{v} h_{A B}(r=2 M, v, \Theta)=-4 M\left(D_{A} D_{B} \hat{C}(\Theta)-\frac{1}{2} \gamma_{A B} D^{2} \hat{C}\right) .
$$

At the quantum level, the expectation value of the metric perturbation in the semiclassical state produced by the shock wave must have the profile (5.15). That is, it must be close to a coherent state of soft gravitons. According to Weinberg's theorem, soft gravitons at $\mathcal{I}^{+}$ are excited whenever energy crosses $\mathcal{I}^{+}$with an $\ell>1$ angular momentum profile. Similarly, soft gravitons at $\mathcal{H}^{+}$are excited whenever energy is thrown into the black hole with an $\ell>1$ angular momentum profile. It would be interesting to see this diagrammatically in perturbation theory around Schwarzschild from the emergence of a pole in the soft limit of gravitons falling into the black hole. It may also be possible to use (5.15) to define a 'black hole memory effect' which can be measured by observers hovering just outside the horizon.

It is trivial to generalize this construction to a black hole formed from the vacuum via a Vaidya shock wave at $v=v_{S}$. One simply replaces the mass aspect appearing in (5.10) by

$$
m=M \theta\left(v-v_{S}\right)+\mu \theta\left(v-v_{0}\right) .
$$

Hence hairy black holes can be classically produced from the vacuum. In the next section we see how they are classically distinguished by their superrotation charges.

\section{Classical superrotation charges of supertranslation hair}

Supertranslating a black hole does not add supertranslation charges to the black hole, just as an ordinary translation of a black hole does not add momentum. This follows from the fact that the supertranslation group is abelian, and may also be seen directly by evaluating the charge expressions of the previous section. However, as supertranslations and superrotations do not commute, a supertranslated black hole can and does carry superrotation charges, already at the classical level. In this section we work out these charges for linearly supertranslated Schwarzschild.

From (2.17) the conserved superrotation charges are

$$
\hat{Q}_{Y}^{-}=\frac{1}{8 \pi} \int_{\mathcal{I}_{+}^{-}} d^{2} \Theta \sqrt{\gamma} Y^{A} N_{A}
$$


where $Y^{A}$ is any smooth vector field on the sphere. We are interested in the differential superrotation charges carried by an infinitesimally supertranslated Schwarzschild black hole of the type considered in the previous section. As seen from (4.6), under a supertranslation $\delta_{f} g_{a b}=\mathcal{L}_{f} g_{a b}$ of Schwarzschild

$$
\delta_{f} N_{A}=-3 M \partial_{A} f
$$

It follows immediately that

$$
\hat{Q}_{Y}^{-}\left(g, h=\delta_{f} g\right)=-\frac{3}{8 \pi} \int_{\mathcal{I}_{+}^{-}} d^{2} \Theta \sqrt{\gamma} Y^{A} M \partial_{A} f .
$$

This is nonzero for a generic vector field $Y^{A}$ and supertranslation $f$. An infinite number of superrotation charges can be independently added to the black hole by different choices of $f$. Hence the superrotation charges classically distinguish differently supertranslated black holes. Classical black holes sport an infinite head of "supertranslation hair" which is rearranged essentially every time something is thrown into it. ${ }^{16}$

The most easily measurable quantity is the difference in superrotation charges before and after the supertranslation hair implant. This is because the definition of superrotation charges (including angular momentum) is ambiguous up to conjugation by supertranslations. In particular, in this example, we could conjugate the superrotation charges by $f$ so that all vanish post-implant. However, the pre-implant charges then become nonzero while the difference of pre-post implant charges is unaffected. This is analogous to the standard gravitational memory effect at $\mathcal{I}^{+}$which also measures differences in supertranslation frames.

However one should not conclude from this that only the charge difference is physical, any more than one should conclude that only black hole energy or momentum differences (a special case) are physical. Measurement of absolute (rather than relative) energy, momentum, angular momentum or any of the superrotation charges is also possible but requires specification of an asymptotic Poincare frame. In the physical phase space, two black hole spacetimes which differ by any element of BMS correspond to different points. For the case of boosts, the two spacetimes have different energy. For supertranslations they are energetically degenerate, but carry different superrotation charges (including angular momentum) and are still physically distinct points. In the quantum theory, the corresponding states are orthogonal and can be superposed. An important difference between boosts and supertranslations is that the latter act nontrivially on all the zero-energy vacua as well, ${ }^{17}$ imparting superrotation charges at quadratic order [34]. Hence the phase space of asymptotically flat geometries with nonzero energy and 4 Killing vectors is not a simple product of vacuum and black hole phase spaces. ${ }^{18}$

\footnotetext{
${ }^{16}$ Similar observations were made in [27] and in the context of the membrane paradigm in [30, 44].

${ }^{17}$ Any given boost element of BMS acts nontrivially on a generic vacuum, but every vacuum is preserved by some Poincare subgroup of BMS. There is no preferred Poincare subgroup [93, 94].

${ }^{18}$ As we shall see in the next section, the linearized supertranslation charge around a black hole geometry naturally decomposes into the sum of a horizon term and a $\mathcal{I}$ term, which are not separately conserved in the general nonlinear context. It may be interesting to consider the phase space action of only one term, but such configurations will generically not be static or have Killing symmetries.
} 
The formula (6.3) of superrotation charges requires only the asymptotic behavior of the black hole, and would in a sense pertain to essentially any configuration with the same mass. To understand this, let us suppose we sent the supertranslating shock wave into a star or a collection of stars instead of into a black hole. The wave will excite and rearrange the interior structure of the star and, in the case of multiple stars, their relative motions. Generically gravitational radiation will carry some, but not all, of the superrotation charge back out to infinity, while some will be retained by the star(s). It is unsurprising that a star, or a collection of stars, which has many internal degrees of freedom and possible interior states, can carry many superrotation charges. There is no no-hair theorem for a star. Now consider instead replacing the black hole by a massive stable 'hairless' elementary particle with no internal degrees of freedom. Such an object cannot carry arbitrary superrotation charges: the pre- and post- superrotation charges are generically the same (except for the $\ell=1$ component). To leading order, the supertranslating shock wave will simply be reflected through the origin and scatter back up to future null infinity. The elementary particle has no mechanism to absorb all the superrotation charges. The outgoing wave will cancel the superrotation charges induced by the ingoing wave and, in the far future, the superrotation charges will revert to their initial incoming values.

So we see that in this sense black holes act more like a complex star with many internal degrees of freedom than a massive elementary particle. The observer at infinity can confirm this by sending in shock waves and watching what comes out.

At the same time, we note that the exact definition of supertranslation hair in the nonlinear theory given here relies on the existence of an asymptotically flat spacetime boundary and so is not fully intrinsic to the black hole. Although it may be possible, we do not know how to canonically associate supertranslation hair to a classical stationary black hole in AdS (this is likely related to the discussion in [81]), while a star in AdS clearly retains many internal degrees of freedom. The next section sheds some light on this issue by giving an intrinsic definition of the horizon contribution to the supertranslation charge to linear order around Schwarzschild.

\section{Horizon charges}

In the absence of eternal black holes or massive fields, the linearized supertranslation charges $\hat{Q}_{f}^{+}$can be written as volume integrals of local operators over $\mathcal{I}^{+}$, as explicitly demonstrated in [3]. However for Schwarzschild this is clearly impossible, as $\mathcal{I}^{+}$is not a Cauchy surface. Rather, in the absence of massive fields, $\mathcal{I}^{+} \cup \mathcal{H}^{+}$is a Cauchy surface. Hence one expects a relation of the form

$$
\hat{Q}_{f}^{+}=\hat{Q}_{f}^{\mathcal{I}^{+}}+\hat{Q}_{f}^{\mathcal{H}^{+}} .
$$

The precise form of the horizon contribution $\hat{Q}_{f}^{\mathcal{H}}{ }^{+}$will depend on the coordinate choice used to extend the supertranslations in from the boundary to the horizon. Here we use Bondi coordinates for this purpose. We gauge fix linearized metric fluctuations of the horizon to obtain a physical horizon phase space $\Gamma_{\mathcal{H}^{+}}$. The symplectic form is then constructed and inverted to obtain the Dirac bracket. An expression for $\hat{Q}_{f}^{\mathcal{H}^{+}}$is derived and shown 
to generate supertranslations on $\Gamma_{\mathcal{H}^{+}}$. The construction requires that $\hat{Q}_{f}^{\mathcal{H}^{+}}$and the soft graviton modes, which are nonvanishing on the boundaries $\mathcal{H}_{ \pm}^{+}$of $\mathcal{H}^{+}$, be incorporated as symplectic partners within $\Gamma_{\mathcal{H}^{+}}$.

Our construction of course makes sense only in (leading order) perturbation theory: in the general case the classical horizon is defined only nonlocally and, even worse, in the quantum case it evaporates. It is doubtful that in the presence of interactions a clean separation can be made between the two terms on the right hand side of (7.1). Nevertheless we hope it may prove useful in developing intuition for the effects of supertranslation charge conservation on black hole dynamics.

This section relies heavily on general formulae from the literature [117-119, 132-135] on the covariant canonical formalism and symplectic forms in gravity.

\subsection{Symplectic forms and linearized charges}

We expand in variations $\delta g_{a b}=h_{a b}$ around a fixed background metric $g_{a b}$ which solves the Einstein equation. The variations are taken to obey the linearized vacuum Einstein equation

$$
\square h_{a b}+2 R_{a c b d} h^{c d}-2 R_{c(a} h_{b)}^{c}-\nabla_{a} \nabla_{c} h_{b}^{c}-\nabla_{b} \nabla_{c} h_{a}^{c}+\nabla_{a} \nabla_{b} h=0 .
$$

and so are tangent to the phase space. The general results of [134] give the pre-symplectic structure as

$$
\omega\left(h, h^{\prime}\right)=\int_{\Sigma} * J\left(h, h^{\prime}\right),
$$

where the surface $\Sigma$ is a Cauchy surface in the spacetime and $J$ is the pre-symplectic one-form. Explicitly,

$$
J=\frac{\sqrt{-g}}{16 \pi}\left[\frac{1}{2} h \nabla^{b} h_{a b}^{\prime}-\frac{1}{2} h \nabla_{a} h^{\prime}+\frac{1}{2} h_{a b} \nabla^{b} h^{\prime}-h^{b c} \nabla_{b} h_{a c}^{\prime}+\frac{1}{2} h^{b c} \nabla_{a} h_{b c}^{\prime}-\left(h \leftrightarrow h^{\prime}\right)\right] d x^{a} .
$$

It may be shown that (7.2) implies

$$
d * J=0
$$

Hence $\omega\left(h, h^{\prime}\right)$ is a functional of the background metric and the two on-shell variations $h$ and $h^{\prime}$. It is invariant under deformations of the surface $\Sigma$ which leave the boundary $\partial \Sigma$ fixed.

We are particularly interested in the case where one of the variations - say $h^{\prime}$ - is pure gauge i.e. $h_{a b}^{\prime}=\nabla_{a} \zeta_{b}+\nabla_{b} \zeta_{a} \equiv h_{a b}^{\zeta} . J$ then becomes coexact

$$
* J=\frac{1}{16 \pi} d * F
$$

where $F_{a b}$ is given by

$$
\begin{aligned}
F_{a b}= & \frac{1}{2}\left(\nabla_{a} \zeta_{b}-\nabla_{b} \zeta_{a}\right) h+\left(\nabla_{a} h_{b}^{c}-\nabla_{b} h_{a}^{c}\right) \zeta_{c}+\left(\nabla_{c} \zeta_{a} h_{b}^{c}-\nabla_{c} \zeta_{b} h_{a}^{c}\right) \\
& -\left(\nabla_{c} h_{b}^{c} \zeta_{a}-\nabla_{c} h^{c}{ }_{a} \zeta_{b}\right)-\left(\nabla_{a} h \zeta_{b}-\nabla_{b} h \zeta_{a}\right) .
\end{aligned}
$$


One then has [117-119, 133-135]

$$
\omega\left(h, h^{\zeta}\right)=-\frac{1}{16 \pi} \int_{\partial \Sigma} * F \equiv \hat{Q}_{\zeta}^{\partial \Sigma}
$$

When $\Sigma$ is a Cauchy surface, and this is nonzero, it indicates that the diffeomorphism $\zeta$ acts non-trivially on the physical phase space. $\hat{Q}_{\zeta}^{\partial \Sigma}$ is a conserved charge associated to the surface $\partial \Sigma$ and diffeomorphism $\zeta$ in the sense that it does not depend on the choice of an interior surface $\Sigma$. We use the hat notation $\hat{Q}$ to emphasize that this is the linearized difference in the charge between the geometries $g$ and $g+h$. We will not in this paper consider the integration to finite charges.

In general, the pre-symplectic form $\omega$ has zero modes and cannot be inverted. These are eliminated by imposition of the constraints and a judicious choice of gauge. Once this has been accomplished, the restriction of the pre-symplectic form to the physical subspace is the symplectic form. Since all of the zero modes have now been eliminated, it may be inverted to find the Dirac bracket. Once we have done this, we may decompose a tangent vector in this physical phase space by $h_{a b}=\sum_{i} h^{i} h_{i a b}$ where the tangent index $i$ runs over the (infinite) dimension of the phase space. Defining the inverse of $\omega$ by

$$
\omega^{i j} \omega_{j k}=\delta_{k}^{i}
$$

the Dirac bracket is

$$
\left\{h^{i}, h^{j}\right\}=\omega^{i j}
$$

Using

$$
\hat{Q}_{\zeta}^{\partial \Sigma}=\left(h^{\zeta}\right)^{i} \omega_{i j} h^{j}
$$

it follows immediately that

$$
\left\{\hat{Q}_{\zeta}^{\partial \Sigma}, h^{i}\right\}=\left(h^{\zeta}\right)^{i}
$$

In other words, $\hat{Q}_{\zeta}^{\partial \Sigma}$ infinitesimally generates the action of the symmetry $\zeta$ on the physical phase space via the Dirac bracket.

\subsection{Schwarzschild charges}

We work in the Bondi gauge (2.1) for which

$$
h_{r r}=h_{r A}=\gamma^{A B} h_{A B}=0,
$$

and are interested in the case $\partial \Sigma$ is an $S^{2}$ of constant $r$ and $v$. One then has

$$
\hat{Q}_{\zeta}^{\partial \Sigma}=-\frac{1}{16 \pi} \int_{\partial \Sigma} d^{2} \Theta \sqrt{\gamma} r^{2} F_{r v}
$$

where for Schwarzschild the general expression (7.7) reduces to

$$
\begin{aligned}
F_{r v}= & \zeta^{A}\left(\partial_{r} h_{A v}-\frac{2}{r} h_{A v}\right)+\zeta^{v}\left(-\frac{1}{r^{2}} D^{A} h_{A v}-\frac{2}{r} h_{v v}\right)+\partial_{r} \zeta^{v} h_{v v}+\frac{1}{r^{2}} D^{A} \zeta^{v} h_{v A} \\
& +\partial_{r} \zeta^{v} V h_{v r}-\zeta^{v} \frac{4 V}{r} h_{v r}+\zeta^{r} \frac{2}{r} h_{v r} .
\end{aligned}
$$


For $\zeta=\zeta_{f}$ a supertranslation as given in (4.5), after discarding total derivatives on $S^{2}$, we find

$$
F_{r v}=\frac{1}{r} D^{A} f \partial_{r} h_{A v}-f\left(\frac{2}{r} h_{v v}+\frac{4 V}{r} h_{v r}\right)-D^{2} f \frac{1}{r} h_{v r} .
$$

Given the large $r$ asymptotics, only the $h_{v v}$ term survives for $r \rightarrow \infty$ and one finds

$$
\hat{Q}_{\zeta_{f}}^{\mathcal{I}_{+}^{-}}=\frac{1}{4 \pi} \int_{\mathcal{I}_{+}^{-}} d^{2} \Theta \sqrt{\gamma} f \hat{m}
$$

which is the standard expression for the incoming linearized supertranslation charge $\hat{Q}_{f}^{-}$. The hat on $\hat{m}$ denotes that it is the deviation of the Bondi mass aspect from the background around which we are expanding. Supertranslation charge conservation equates this to the outgoing charge

$$
\hat{Q}_{f}^{\mathcal{I}_{+}^{-}}=\hat{Q}_{f}^{\mathcal{I}_{-}^{+}}=\frac{1}{4 \pi} \int_{\mathcal{I}_{-}^{+}} d^{2} \Theta \sqrt{\gamma} f \hat{m}
$$

Assuming there are no massive particles ${ }^{19}$ or black holes, after imposing constraints and fixing to the Bondi gauge, the symplectic form (7.3) was inverted in [3] to obtain the Dirac bracket $\{$,$\} . Using the constraints to rewrite the charge as a \mathcal{I}^{-}$integral

$$
\hat{Q}_{f}^{\mathcal{I}^{-}}=\hat{Q}_{f}^{\mathcal{I}_{+}^{-}}=\frac{1}{4 \pi} \int_{\mathcal{I}^{-}} d^{2} \Theta d v \sqrt{\gamma} f\left(T_{v v}-\frac{1}{4} D^{A} D^{B} N_{A B}\right) .
$$

It was then shown that on $\mathcal{I}^{-}$

$$
\left\{\hat{Q}_{f}^{-},\right\}=\delta_{f}
$$

That is, in this special case the supertranslation charge indeed generates supertranslations via the Dirac bracket.

We would like to preform a similar construction of the charge in the presence of a black hole. Let $\mathcal{X}^{+}$be a hypersurface extending from $\mathcal{I}_{-}^{+}$to $\mathcal{H}_{+}^{+}$, so that $\mathcal{X}^{+} \cup \mathcal{H}^{+}$is a Cauchy surface for the portion of the spacetime exterior to the black hole. Then ${ }^{20}$

$$
\hat{Q}_{f}^{-}=\hat{Q}_{f}^{\mathcal{X}^{+}}+\hat{Q}_{f}^{\mathcal{H}^{+}}
$$

$\hat{Q}_{f}^{\mathcal{H}^{+}}$is the contribution to the full supertranslation charge coming from the horizon: i.e. the black hole supertranslation charge. It is the difference between two boundary terms

$$
\hat{Q}_{f}^{\mathcal{H}^{+}}=\frac{M}{8 \pi} \int d^{2} \Theta \sqrt{\gamma} f\left[D^{A} \partial_{r} h_{A v}+2 h_{v v}+D^{2} h_{v r}\right]_{\mathcal{H}_{-}^{+}}^{\mathcal{H}_{+}^{+}} .
$$

We wish to study its properties and demonstrate that it generates horizon supertranslations.

\footnotetext{
${ }^{19}$ The additional boundary terms at $\mathcal{I}_{-}^{-}$from massive fields are given in [11].

${ }^{20}$ For eternal Schwarzschild, which has a past horizon, charge conservation will require a matching condition relating data on $\mathcal{H}_{-}^{+}$to that on $\mathcal{H}_{+}^{-}$.
} 
$\hat{Q}_{f}^{\mathcal{H}+}$ can be written as a horizon integral by integrating by parts and using the constraints. The linearized constraints on the horizon are ${ }^{21}$

$$
\begin{gathered}
\partial_{v}\left(D^{A} h_{A v}+2 M h_{v v}\right)-\frac{1}{4 M} D^{A} h_{A v}-\frac{1}{2} D^{2} h_{v v}=32 \pi M^{2} T_{v v}^{M} . \\
\partial_{v}\left(-D_{A} h_{v r}-\partial_{r} h_{A v}+\frac{1}{M} h_{v A}+\frac{1}{4 M^{2}} D^{B} h_{B A}\right)+D_{A} \partial_{r} h_{v v}+\frac{1}{2 M} D_{A} h_{v r} \\
+\frac{1}{4 M^{2}} D_{A} D^{B} h_{B v}-\frac{1}{4 M^{2}} D^{2} h_{A v}-\frac{1}{4 M^{2}} h_{A v}=16 \pi T_{A v}^{M} .
\end{gathered}
$$

Using the linear combination of (7.23) and the divergence of (7.24) given by

$$
\begin{array}{r}
M \partial_{v}\left(2 h_{v v}+D^{2} h_{v r}+D^{A} \partial_{r} h_{A v}\right)-\frac{1}{2} D^{2} h_{v v}-\frac{1}{4 M} D^{A} D^{B} \partial_{v} h_{B A}-M D^{2} \partial_{r} h_{v v} \\
-\frac{1}{2} D^{2} h_{v r}+\frac{1}{4 M} D^{A} h_{A v}=32 \pi M^{2} T_{v v}^{M}-16 \pi M D^{A} T_{A v}^{M}
\end{array}
$$

and integrating by parts it finally follows that

$$
\begin{aligned}
\hat{Q}_{f}^{\mathcal{H}^{+}}=\frac{1}{8 \pi} \int_{\mathcal{H}^{+}} d^{2} \Theta & \sqrt{\gamma} d v f\left(\frac{1}{4 M} D^{A} D^{B} \partial_{v} h_{B A}+32 \pi M^{2} T_{v v}^{M}-16 \pi M D^{A} T_{A v}^{M}\right. \\
+ & \left.\frac{1}{2} D^{2} h_{v v}+M D^{2} \partial_{r} h_{v v}+\frac{1}{2} D^{2} h_{v r}-\frac{1}{4 M} D^{A} h_{A v}\right) .
\end{aligned}
$$

We will see that this generates horizon supertranslations after appropriate gauge fixing and boundary conditions.

\subsection{Gauge fixing and Dirac brackets}

We cannot yet construct Dirac brackets because the presymplectic form $\omega$ still has zero eigenvectors given by residual gauge transformations which vanish at $\mathcal{H}_{ \pm}^{+}$and preserve the Bondi gauge (4.2)-(4.4). We now find the most general such transformation. Differentiating (4.2) with respect to $r$ and using (4.3) one finds the condition

$$
r \partial_{r}^{2} \zeta^{A}+2 \partial_{r} \zeta^{A}=0
$$

The general solution to the above equation is

$$
\zeta^{A}(r, v, \Theta)=X^{A}(v, \Theta)+\frac{1}{r} Z^{A}(v, \Theta) .
$$

Substituting $\zeta^{A}(r, v, \Theta)$ from above into (4.2)-(4.4) gives the remaining components of $\zeta^{a}$,

$$
\zeta^{r}=-\frac{r}{2} D_{A} \zeta^{A}, \quad \partial_{A} \zeta^{v}=\gamma_{A B} Z^{B}
$$

Let us define $\zeta^{v}=X(v, \Theta)$. Then the most general residual diffeomorphism $\zeta_{X}$ for Schwarzschild in Bondi gauge ${ }^{22}$ is parametrized by an arbitrary vector $X^{A}(v, \Theta)$ and an arbitrary scalar $X(v, \Theta)$ on $\mathcal{H}^{+}$as:

$$
\zeta_{X}=X \partial_{v}-\frac{1}{2}\left(r D_{A} X^{A}+D^{2} X\right) \partial_{r}+X^{A} \partial_{A}+\frac{1}{r} D^{A} X \partial_{A}
$$

\footnotetext{
${ }^{21}$ In this section consider only linearized order where the matter stress tensor $T^{M}$ vanishes, we include it here only as an indicator of how matter couples at next order.

${ }^{22}$ This is more general than the usual BMS vector fields discussed e.g. in [85-88] as we have not imposed any falloffs.
} 
These shift the nonzero metric perturbations to leading order as follows

$$
\begin{aligned}
\delta_{X} h_{v v} & =\frac{M}{r} D_{B} X^{B}+\frac{M}{r^{2}} D^{2} X-2 V \partial_{v} X-r \partial_{v} D_{B} X^{B}-D^{2} \partial_{v} X \\
\delta_{X} h_{A v} & =-\frac{r}{2} D_{A} D_{B} X^{B}-\frac{1}{2} D_{A} D^{2} X-V \partial_{A} X+r^{2} \partial_{v} X_{A}+r \partial_{v} D_{A} X \\
\delta_{X} h_{A B} & =r^{2}\left(D_{A} X_{B}+D_{B} X_{A}-\gamma_{A B} D_{C} X^{C}\right)+r\left(2 D_{A} D_{B} X-\gamma_{A B} D^{2} X\right), \\
\delta_{X} h_{v r} & =-\frac{1}{2} D_{B} X^{B}+\partial_{v} X
\end{aligned}
$$

where, as usual, $X_{A}=\gamma_{A B} X^{B}$ and $\delta_{X}$ denotes the Lie action of $\zeta_{X}$ on Schwarzschild. A supertranslation is

$$
X=f, \quad X^{A}=0,
$$

with $\partial_{v} f=0$, while a superrotation is ${ }^{23}$

$$
X=\frac{v}{2} D_{A} Y^{A}, \quad X^{A}=Y^{A},
$$

with $\partial_{v} Y^{A}=0$.

In order to invert the symplectic form to get the Dirac bracket we must fix the trivial gauge symmetry, namely those transformations which (unlike supertranslations) are zero eigenvectors of $\omega$ and then use the Einstein equation to restrict to the 'on-shell' physical phase space. We moreover by hand restrict the phase space via the boundary condition $\left.\partial_{v} h_{a b}\right|_{v= \pm \infty}=0$. This excludes the superrotations (7.33): a looser boundary condition (see $[45,121,124,125])$ is certainly of interest but outside our current scope. Having done so, we will find $\omega$ in two steps. First we will construct a reduced $\omega^{\text {red }}$ in which all gauge freedom is eliminated. We will then add in the non-trivial gauge modes which requires only the boundary expression (7.15).

The constraints (7.23), (7.24) imply that the linear combinations

$$
\begin{array}{r}
{\left[\frac{1}{4 M} D^{A} h_{A v}+\frac{1}{2} D^{2} h_{v v}\right]_{\mathcal{H}_{ \pm}^{+}}=0,} \\
{\left[D_{A} \partial_{r} h_{v v}+\frac{1}{2 M} D_{A} h_{v r}+\frac{1}{4 M^{2}} D_{A} D^{B} h_{B v}-\frac{1}{4 M^{2}} D^{2} h_{A v}-\frac{1}{4 M^{2}} h_{A v}\right]_{\mathcal{H}_{ \pm}^{+}}=0,}
\end{array}
$$

vanish at the horizon boundaries $\mathcal{H}_{ \pm}^{+}$where we have set $\partial_{v} h_{a b}=0$. Defining two convenient combinations of the metric perturbations $\tilde{h}$ and $\tilde{h}_{A}$ by

$$
\begin{aligned}
\tilde{h} & \equiv h_{v r}+2 h_{v v}+2 M \partial_{r} h_{v v}, \\
\tilde{h}_{A} & \equiv h_{A v}+2 M D_{A} h_{v v}
\end{aligned}
$$

\footnotetext{
${ }^{23}$ Locally imposing the standard Bondi falloff conditions at large $r$ requires $Y^{A}$ to be locally a conformal Killing vector [85-88] and implies $D^{2} D_{A} Y^{A}=-2 D_{A} Y^{A}$. We will not impose this restriction herein. The general expression for the charge is $\hat{Q}_{\left(X, X^{A}\right)}=-\frac{1}{16 \pi} \int_{\partial \Sigma} d^{2} \Theta \sqrt{\gamma} r\left[X^{A}\left(r \partial_{r} h_{A v}-\right.\right.$ $\left.\left.2 h_{A v}+r D^{A} h_{v r}\right)-X\left(D^{A} \partial_{r} h_{A v}+2 h_{v v}+\left(D^{2}+4 V\right) h_{v r}\right)\right]$, while the central term is $\omega\left(X, X^{A} ; X^{\prime}, X^{\prime A}\right)=$ $\frac{1}{16 \pi} \int_{\partial \Sigma} d^{2} \Theta \sqrt{\gamma} r\left[X\left(-D^{2}-2+\frac{6 M}{r}\right) D_{A} X^{\prime A}-\left(X \rightarrow X^{\prime}, X^{\prime A} \rightarrow X^{A}\right)\right]$.
} 
(7.34) and (7.35) can be rewritten

$$
\begin{aligned}
\left.D^{A} \tilde{h}_{A}\right|_{\mathcal{H}_{ \pm}^{+}} & =0, \\
{\left[\left(D^{2}+1\right) \tilde{h}_{A}-2 M D_{A} \tilde{h}\right]_{\mathcal{H}_{ \pm}^{+}} } & =0 .
\end{aligned}
$$

These equations have angular momentum $\ell=0,1$ solutions with $\tilde{h}_{A}$ a rotational Killing vector and $\tilde{h}$ a constant on the sphere. These are related to linearized deformations of the angular momentum and mass of the black hole which are not our present interest. While inclusion of these four modes would not change our final conclusions for simplicity we fix them to zero:

$$
\left.\tilde{h}_{A}\right|_{\mathcal{H}_{ \pm}^{+}}=\left.\tilde{h}\right|_{\mathcal{H}_{ \pm}^{+}}=0 .
$$

Under the general residual $X$ and $X^{A}$ gauge transformations (7.31) one has

$$
\begin{aligned}
\delta_{X} \tilde{h} & =-\left(2 D^{2}+1\right) \partial_{v} X-6 M \partial_{v} D_{B} X^{B}, \\
\delta_{X} \tilde{h}_{A} & =4 M^{2}\left(\partial_{v} X_{A}-\partial_{v} D_{A} D_{B} X^{B}\right)-2 M D_{A}\left(D^{2}-1\right) \partial_{v} X .
\end{aligned}
$$

We can use these to set $($ for $\ell \neq 0,1)$ )

$$
\tilde{h}=\tilde{h}_{a}=0
$$

everywhere on the horizon $r=2 M$. This still leaves unfixed all $\partial_{v} X=\partial_{v} X^{A}=0$ transformations, which includes supertranslations. Using (7.43) to eliminate $h_{v r}$ and $h_{A v}$, the constraints simplify to

$$
\begin{aligned}
\left(D^{2}-1\right) \partial_{v}\left[h_{v v}\right]=-16 \pi M T_{v v}^{M} & =0, \\
\partial_{v}\left[2 M D_{A} \partial_{r} h_{v v}-\partial_{r} h_{A v}+\frac{1}{4 M^{2}} D^{B} h_{B A}\right]=16 \pi T_{A v}^{M} & =0 .
\end{aligned}
$$

The matter sources are set to zero here because we are constructing the Dirac brackets of the linearized theory. We conclude the quantities in square brackets are function of $\Theta$ only. Using the residual $\partial_{v} X=\partial_{v} X^{A}=0$ symmetry we may set then to 0 :

$$
\begin{aligned}
h_{v v} & =0, \\
\partial_{r} h_{A v} & =2 M D_{A} \partial_{r} h_{v v}+\frac{1}{4 M^{2}} D^{B} h_{B A} .
\end{aligned}
$$

It can be shown that this completely fixes all the gauge symmetry, including supertranslations. Using these relations, the $r r$ and $r v$ components of the Einstein equations reduce to

$$
\begin{aligned}
\frac{1}{M} \partial_{r} h_{v r} & =8 \pi T_{r r}^{M}=0, \\
-\frac{1}{2 M}\left(D^{2}-1\right) \partial_{r} h_{v v}-\frac{1}{16 M^{4}} D^{A} D^{B} h_{A B} & =8 \pi T_{v r}^{M}=0 .
\end{aligned}
$$

The symplectic form (7.3) involves $\left(h_{A B}, h_{v v}, \partial_{r} h_{v v}, h_{r v}, \partial_{r} h_{r v}, h_{A v}, \partial_{r} h_{A v}\right)$. (7.43) can be used to eliminate $h_{A v}$ and $h_{v r}$ in terms of other variables. (7.46)-(7.49) then eliminate 
$h_{v v}, \partial_{r} h_{A v}, \partial_{r} h_{v r}$ and $\partial_{r} h_{v v}$, expressing everything in terms of the traceless $h_{A B}$. Denoting such fully gauge-fixed on-shell perturbations by $\bar{h}_{a b}$, one finds

$$
\begin{aligned}
\bar{h}_{v v} & =0, \\
\partial_{r} \bar{h}_{v v} & =-\frac{1}{8 M^{3}}\left[D^{2}-1\right]^{-1} D^{A} D^{B} \bar{h}_{A B}, \\
\bar{h}_{v r} & =\frac{1}{4 M^{2}}\left[D^{2}-1\right]^{-1} D^{A} D^{B} \bar{h}_{A B}, \\
\bar{h}_{A v} & =0, \\
\partial_{r} \bar{h}_{A v} & =-\frac{1}{4 M^{2}} D_{A}\left[D^{2}-1\right]^{-1} D^{B} D^{C} \bar{h}_{B C}+\frac{1}{4 M^{2}} D^{B} \bar{h}_{A B}, \\
\partial_{r} \bar{h}_{v r} & =0 .
\end{aligned}
$$

It should be noted that $\left(D^{2}-1\right)$ is a negative definite operator and therefore its inverse exists.

A computation reveals that

$$
\omega^{r e d}\left(\bar{h}, \bar{h}^{\prime}\right)=\frac{1}{64 \pi M^{2}} \int d v d^{2} \Theta \sqrt{\gamma}\left[\bar{h}^{A B} \sigma_{A B}^{\prime}-\bar{h} \leftrightarrow \bar{h}^{\prime}\right]_{\mathcal{H}_{-}^{+}}^{\mathcal{H}_{+}^{+}} .
$$

Here the traceless shear tensor

$$
\sigma_{A B}=\frac{1}{2} \partial_{v} h_{A B}
$$

is the local coordinate-invariant dynamical degree of freedom on the horizon. (7.50) expresses the fact that after complete gauge-fixing and imposition of the constraints all metric components are determined by the shear tensor, up to zero modes. It is of course the point of this paper to carefully understand the zero modes.

At the level of linearized metric perturbations around Schwarzschild, it appears self consistent to view $\bar{h}_{a b}$ as a complete set of coordinates on the phase space of the horizon. However as we have seen in section 5, the moment interactions are introduced, pure gauge modes corresponding to supertranslations are excited. The gauge condition (7.46), which eliminated the rigid supertranslations cannot be enforced. Hence one cannot perturbatively construct the interacting theory beginning from fully gauge fixed modes. One must, at a minimum introduce the supertranslation field $\delta_{f} g_{a b}$, which we shall see shortly is not a zero mode of the presymplectic form $\omega .^{24}$ The fully gauge fixed perturbation $\bar{h}_{a b}$ is related to the more general Bondi-gauge perturbation $h_{a b}$ by

$$
h_{a b}=\bar{h}_{a b}+\delta_{f} g_{a b}
$$

The full symplectic form is

$$
\omega^{r e d}\left(h, h^{\prime}\right)=\omega\left(\bar{h}, \bar{h}^{\prime}\right)+\omega^{r e d}\left(\bar{h}, \delta_{f^{\prime}} g\right)+\omega^{r e d}\left(\delta_{f} g, \bar{h}^{\prime}\right)+\omega^{r e d}\left(\delta_{f} g, \delta_{f^{\prime}} g\right) .
$$

The last term is easily seen to vanish, implying there is no classical central term in the supertranslation algebra. The middle two terms were essentially computed in (7.26). Using (7.43) and setting the sources to zero, one finds

$$
\omega^{r e d}\left(h, \delta_{f^{\prime}} g\right)=\frac{1}{16 \pi M} \int_{\mathcal{H}^{+}} d v d^{2} \Theta \sqrt{\gamma} f^{\prime} D^{A} D^{B} \sigma_{A B}
$$

\footnotetext{
${ }^{24}$ Other pure gauge modes which are not annihilated by the symplectic form are interesting candidates for further physical degrees of freedom, but are beyond the scope of the present paper.
} 
Putting this together and using $h_{A B}=\bar{h}_{A B}+2 M\left(2 D_{A} D_{B} f-\gamma_{A B} D^{2} f\right)$, one finds

$$
\omega^{r e d}\left(h, h^{\prime}\right)=\frac{1}{64 \pi M^{2}} \int d^{2} \Theta \sqrt{\gamma} \int d v h^{A B} \sigma_{A B}^{\prime}-h \leftrightarrow h^{\prime},
$$

This implies the Dirac bracket

$$
\left\{\sigma_{A B}(\Theta, v), h_{C D}\left(\Theta^{\prime}, v^{\prime}\right)\right\}=32 \pi M^{2}\left(\gamma_{A C} \gamma_{B D}+\gamma_{A D} \gamma_{B C}-\gamma_{A B} \gamma_{C D}\right) \delta\left(v-v^{\prime}\right) \delta^{2}\left(\Theta-\Theta^{\prime}\right),
$$

The expression in parentheses is the deWitt metric [136] for computing distances on the space of all metrics. Hence

$$
\hat{Q}_{f}^{\mathcal{H}^{+}}=\frac{1}{16 \pi M} \int_{\mathcal{H}^{+}} d v d^{2} \Theta \sqrt{\gamma} f D^{A} D^{B} \sigma_{A B}
$$

and

$$
\left\{\hat{Q}_{f}^{\mathcal{H}^{+}}, h_{A B}\right\}=2 M\left(2 D_{A} D_{B} f-\gamma_{A B} D^{2} f\right)
$$

as desired. ${ }^{25}$ This equations state that the linearized charge $\hat{Q}_{f}^{\mathcal{H}^{+}}$is the symplectic partner of the supertranslation zero mode $\delta_{f} g_{a b}$. This was guaranteed to work by the general argument of section 7.1 once the physical phase space and sympectic form were properly identified.

The linearized charge $\hat{Q}_{f}^{\mathcal{H}^{+}}$is a multipole moment of the zero mode of the shear tensor which is the local Cauchy data on the horizon. $\hat{Q}_{f}^{\mathcal{H}^{+}}$does not vanish for generic shear tensor on $\mathcal{H}^{+}$. If it did, it could not generate supertranslations via Dirac brackets on the physical phase space. However, if we look at the space of linearized fluctuations on $\mathcal{H}^{+}$ that can be excited by sending in linearized gravity waves from $\mathcal{I}^{-}$, they all have $\hat{Q}_{f}^{\mathcal{H}^{+}}=0$. This follows from the fact that the black hole absorption amplitude for the $\ell$ th partial wave with frequency $\omega$ is proportional to $\omega^{\ell} .{ }^{26}$ The $\mathcal{H}^{+}$Cauchy data with nonzero $\hat{Q}_{f}^{\mathcal{H}^{+}}$would, if evolved backwards with the linearized equations, give perturbations which diverge far from $\mathcal{H}^{+}$on $\mathcal{I}^{-}$. Nevertheless, we here see that the horizon phase space parametrized by $\bar{h}_{A B}$ must be enlarged by the symplectic pair $\left(\hat{Q}_{f}^{\mathcal{H}^{+}}, \delta_{f} g_{a b}\right)$ in order to have a suitable starting point for the interacting theory in which, as we have seen in section 5 , the supertranslation field can not be frozen. Equivalently, the enlargement of the phase space is required for the existence of an operator which generates supertranslations everywhere in the spacetime.

\section{Acknowledgments}

We are grateful to Abhay Ashtekhar, Glenn Barnich, Piotr Chrusciel, Geoffrey Compere, Mihalis Dafermos, Sasha Haco, Dan Kapec, Alex Lupsasca, Prahar Mitra, Monica Pate, David Skinner, Bob Wald and S. T. Yau for useful discussions. This work was supported in part by the Black Hole Initiative, STFC, DOE grant DE-FG02-91ER40654, the George and Cynthia Mitchell Foundation, Trinity College Cambridge, the Templeton Foundation and a Bershadsky Visiting Fellowship to MJP.

\footnotetext{
${ }^{25}$ When interactions are included, the charge acquires terms quadratic in $h$, and the right hand side has homogeneous terms such as $f \partial_{v} h_{A B}$.

${ }^{26}$ This has recently been shown to follow directly from supertranslation charge conservation [23].
} 


\section{A Some useful formulae}

In this appendix we collect some formulae which we have found useful in our computations. In Schwarzschild the nonzero connection coefficients are

$$
\begin{array}{llll}
\Gamma_{r B}^{A}=\frac{\delta_{B}^{A}}{r}, & \Gamma_{A B}^{v}=-r \gamma_{A B}, & \Gamma_{A B}^{r}=-r V \gamma_{A B}, & \Gamma_{B C}^{A}={ }^{(2)} \Gamma_{B C}^{A}, \\
\Gamma_{v r}^{r}=-\frac{M}{r^{2}}, & \Gamma_{v v}^{v}=\frac{M}{r^{2}}, & \Gamma_{v v}^{r}=\frac{M V}{r^{2}} .
\end{array}
$$

Covariant derivatives on the unit sphere obey

$$
\left[D^{2}, D_{A}\right] X=\partial_{A} X, \quad\left[D^{B}, D_{A}\right] X_{B}=X_{A} .
$$

One finds at $r=2 M$

$$
\begin{aligned}
F_{r v}= & \zeta^{A}\left(\partial_{r} h_{A v}-\frac{1}{M} h_{A v}\right)+\zeta^{v}\left(-\frac{1}{4 M^{2}} D^{A} h_{A v}+\frac{1}{4 M^{2}} \partial_{v} h_{A}^{A}-\frac{1}{M} h_{v v}-\frac{1}{16 M^{3}} h_{A}^{A}\right) \\
& +\partial_{r} \zeta^{v} h_{v v}+\frac{1}{4 M^{2}} D^{A} \zeta^{v} h_{v A}-\frac{1}{8 M^{2}} \partial_{v} \zeta^{v} h_{A}^{A}+\zeta^{r}\left(\frac{1}{M} h_{v r}+\frac{1}{8 M^{3}} h_{A}^{A}\right)+\frac{1}{8 M^{2}} \partial_{r} \zeta^{r} h_{A}^{A}
\end{aligned}
$$

On the horizon, supertranslations are given by

$$
\begin{aligned}
\delta_{f} h_{v v} & =\frac{1}{4 M} D^{2} f, \\
\delta_{f} \partial_{r} h_{v v} & =-\frac{1}{4 M^{2}} D^{2} f, \\
\delta_{f} h_{A v} & =-\frac{1}{2} \partial_{A} D^{2} f, \\
\delta_{f} \partial_{r} h_{A v} & =-\frac{1}{2 M} \partial_{A} f, \\
\delta_{f} h_{A B} & =2 M\left(2 D_{A} D_{B} f-\gamma_{A B} D^{2} f\right), \\
D^{2} D_{A} f & =D_{A} D^{2} f+\partial_{A} f
\end{aligned}
$$

From the linearized constraints on the horizon $(7.23)(7.24)$

$$
\begin{aligned}
\partial_{r} h_{v v}=-\frac{1}{4 M} h, \quad h_{v r}=\frac{1}{2} h, \quad \partial_{r} h_{A v} & =-\frac{1}{2} D_{A} h+\frac{1}{4 M^{2}} D^{B} h_{A B}, \quad h_{v v}=h_{A v}=0 . \\
\nabla^{b} h_{b v} & =\frac{1}{2} \partial_{v} h \\
\nabla_{b} h_{v c}-\nabla_{v} h_{b c} & =\partial_{b} h_{v c}-\partial_{v} h_{b c}-\Gamma_{b c}^{a} h_{v a}+\Gamma_{v c}^{a} h_{b a} \\
\nabla_{r} h_{v v}-\nabla_{v} h_{r v} & =-\frac{1}{2} \partial_{v} h, \\
\nabla_{B} h_{v C}-\nabla_{v} h_{B C} & =\partial_{v} h_{B C} .
\end{aligned}
$$

Then the pre-symplectic current becomes

$$
\begin{aligned}
J_{v} & =\frac{\sqrt{-g}}{16 \pi}\left[\frac{1}{4} h \partial_{v} h^{\prime}-\frac{1}{2} h \partial_{v} h^{\prime}+\frac{1}{4} h \partial_{v} h^{\prime} \frac{1}{2} h^{A B} \partial_{v} h_{A B}^{\prime}-h \leftrightarrow h^{\prime}\right] \\
& =\frac{\sqrt{-g}}{32 \pi} h^{A B} \partial_{v} h_{A B}^{\prime}-h \leftrightarrow h^{\prime}
\end{aligned}
$$


Open Access. This article is distributed under the terms of the Creative Commons Attribution License (CC-BY 4.0), which permits any use, distribution and reproduction in any medium, provided the original author(s) and source are credited.

\section{References}

[1] A. Strominger, Asymptotic symmetries of Yang-Mills theory, JHEP 07 (2014) 151 [arXiv:1308.0589] [INSPIRE].

[2] A. Strominger, On BMS invariance of gravitational scattering, JHEP 07 (2014) 152 [arXiv:1312.2229] [INSPIRE].

[3] T. He, V. Lysov, P. Mitra and A. Strominger, BMS supertranslations and Weinberg's soft graviton theorem, JHEP 05 (2015) 151 [arXiv:1401.7026] [INSPIRE].

[4] S. Hyun, S.-A. Park and S.-H. Yi, Quasi-local charges and asymptotic symmetry generators, JHEP 06 (2014) 151 [arXiv: 1403.2196] [INSPIRE].

[5] T. Adamo, E. Casali and D. Skinner, Perturbative gravity at null infinity, Class. Quant. Grav. 31 (2014) 225008 [arXiv:1405.5122] [INSPIRE].

[6] T. He, P. Mitra, A.P. Porfyriadis and A. Strominger, New symmetries of massless QED, JHEP 10 (2014) 112 [arXiv:1407.3789] [INSPIRE].

[7] M. Campiglia and A. Laddha, New symmetries for the gravitational S-matrix, JHEP 04 (2015) 076 [arXiv: 1502.02318] [INSPIRE].

[8] M. Campiglia and A. Laddha, Asymptotic symmetries of QED and Weinberg's soft photon theorem, JHEP 07 (2015) 115 [arXiv: 1505.05346] [INSPIRE].

[9] D. Kapec, M. Pate and A. Strominger, New symmetries of QED, arXiv:1506.02906 [INSPIRE].

[10] S.G. Avery and B.U.W. Schwab, Burg-Metzner-Sachs symmetry, string theory and soft theorems, Phys. Rev. D 93 (2016) 026003 [arXiv: 1506.05789] [InSPIRE].

[11] M. Campiglia and A. Laddha, Asymptotic symmetries of gravity and soft theorems for massive particles, JHEP 12 (2015) 094 [arXiv: 1509.01406] [INSPIRE].

[12] S.G. Avery and B.U.W. Schwab, Residual local supersymmetry and the soft gravitino, Phys. Rev. Lett. 116 (2016) 171601 [arXiv: 1512.02657] [INSPIRE].

[13] V. Lysov, Asymptotic fermionic symmetry from soft gravitino theorem, arXiv:1512.03015 [INSPIRE].

[14] A. Strominger, Infrared structure of gravity and gauge theories, Physics 211r lecture videos online at https://www.youtube.com/playlist?list=PLwLjkVy3evOazQ3FoRH-

Sz8Eoxx2oriXL, Harvard University, U.S.A., Spring 2016.

[15] A. Ashtekar and R.O. Hansen, A unified treatment of null and spatial infinity in general relativity I - universal structure, asymptotic symmetries and conserved quantities at spatial infinity, J. Math. Phys. 19 (1978) 1542 [INSPIRE].

[16] A. Ashtekar, Asymptotic quantization of the gravitational field, Phys. Rev. Lett. 46 (1981) 573 [INSPIRE].

[17] A. Ashtekar and M. Streubel, Symplectic geometry of radiative modes and conserved quantities at null infinity, Proc. Roy. Soc. Lond. A 376 (1981) 585 [InSPIRE]. 
[18] A. Ashtekar, Asymptotic quantization: based on 1984 Naples lectures, Bibliopolis, Naples Italy, (1987) [INSPIRE].

[19] A.P. Balachandran and S. Vaidya, Spontaneous Lorentz violation in gauge theories, Eur. Phys. J. Plus 128 (2013) 118 [arXiv:1302.3406] [inSPIRE].

[20] A. Strominger and A. Zhiboedov, Gravitational memory, BMS supertranslations and soft theorems, JHEP 01 (2016) 086 [arXiv:1411.5745] [INSPIRE].

[21] S. Pasterski, A. Strominger and A. Zhiboedov, New gravitational memories, JHEP 12 (2016) 053 [arXiv: 1502.06120] [INSPIRE].

[22] S.W. Hawking, M.J. Perry and A. Strominger, Soft hair on black holes, Phys. Rev. Lett. 116 (2016) 231301 [arXiv:1601.00921] [INSPIRE].

[23] S.G. Avery and B.U.W. Schwab, Soft black hole absorption rates as conservation laws, JHEP 04 (2017) 053 [arXiv:1609.04397] [INSPIRE].

[24] P.T. Chruściel, J. Lopes Costa and M. Heusler, Stationary black holes: uniqueness and beyond, Living Rev. Rel. 15 (2012) 7 [arXiv:1205.6112] [INSPIRE].

[25] S.W. Hawking, Breakdown of predictability in gravitational collapse, Phys. Rev. D 14 (1976) 2460 [inSPIRE].

[26] D. Kapec, V. Lysov, S. Pasterski and A. Strominger, Semiclassical Virasoro symmetry of the quantum gravity S-matrix, JHEP 08 (2014) 058 [arXiv:1406.3312] [INSPIRE].

[27] É.É. Flanagan and D.A. Nichols, Conserved charges of the extended Bondi-Metzner-Sachs algebra, Phys. Rev. D 95 (2017) 044002 [arXiv:1510.03386] [INSPIRE].

[28] G. Compère and J. Long, Classical static final state of collapse with supertranslation memory, Class. Quant. Grav. 33 (2016) 195001 [arXiv:1602.05197] [INSPIRE].

[29] T. Banks, The super BMS algebra, scattering and holography, arXiv:1403.3420 [INSPIRE].

[30] R.F. Penna, BMS invariance and the membrane paradigm, JHEP 03 (2016) 023 [arXiv: 1508.06577] [INSPIRE].

[31] T.T. Dumitrescu, T. He, P. Mitra and A. Strominger, Infinite-dimensional fermionic symmetry in supersymmetric gauge theories, arXiv:1511.07429 [INSPIRE].

[32] G. 't Hooft, Black hole unitarity and antipodal entanglement, Found. Phys. 46 (2016) 1185 [arXiv: 1601.03447] [INSPIRE].

[33] A. Averin, G. Dvali, C. Gomez and D. Lüst, Gravitational black hole hair from event horizon supertranslations, JHEP 06 (2016) 088 [arXiv: 1601.03725] [INSPIRE].

[34] G. Barnich and C. Troessaert, Finite BMS transformations, JHEP 03 (2016) 167 [arXiv: 1601.04090] [INSPIRE].

[35] G. Compère and J. Long, Vacua of the gravitational field, JHEP 07 (2016) 137 [arXiv: 1601.04958] [INSPIRE].

[36] D.-C. Dai and D. Stojkovic, Pre-Hawking radiation may allow for reconstruction of the mass distribution of the collapsing object, Phys. Lett. B 758 (2016) 429 [arXiv: 1601.07921] [INSPIRE].

[37] A. Campoleoni, H.A. González, B. Oblak and M. Riegler, BMS modules in three dimensions, Int. J. Mod. Phys. A 31 (2016) 1650068 [arXiv:1603.03812] [INSPIRE]. 
[38] H. Afshar et al., Soft Heisenberg hair on black holes in three dimensions, Phys. Rev. D 93 (2016) 101503 [arXiv: 1603.04824] [INSPIRE].

[39] M.M. Sheikh-Jabbari and H. Yavartanoo, On 3d bulk geometry of Virasoro coadjoint orbits: orbit invariant charges and Virasoro hair on locally $A d S_{3}$ geometries,

Eur. Phys. J. C 76 (2016) 493 [arXiv: 1603.05272] [INSPIRE].

[40] M.Z. Iofa, Density matrix of radiation of a black hole with a fluctuating horizon, Phys. Rev. D 94 (2016) 064044 [arXiv:1603.07480] [INSPIRE].

[41] D. Kapec, A.-M. Raclariu and A. Strominger, Area, entanglement entropy and supertranslations at null infinity, arXiv:1603.07706 [INSPIRE].

[42] M.M. Sheikh-Jabbari, Residual diffeomorphisms and symplectic soft hairs: the need to refine strict statement of equivalence principle, Int. J. Mod. Phys. D 25 (2016) 1644019 [arXiv: 1603.07862] [INSPIRE].

[43] A.M. Frassino, S. Köppel and P. Nicolini, Geometric model of black hole quantum $N$-portrait, extradimensions and thermodynamics, Entropy 18 (2016) 181 [arXiv: 1604.03263] [INSPIRE].

[44] C. Eling and Y. Oz, On the membrane paradigm and spontaneous breaking of horizon BMS symmetries, JHEP 07 (2016) 065 [arXiv: 1605.00183] [INSPIRE].

[45] E. Conde and P. Mao, Remarks on asymptotic symmetries and the subleading soft photon theorem, Phys. Rev. D 95 (2017) 021701 [arXiv: 1605.09731] [INSPIRE].

[46] G. Compère, Bulk supertranslation memories: a concept reshaping the vacua and black holes of general relativity, Int. J. Mod. Phys. D 25 (2016) 1644006 [arXiv:1606.00377] [INSPIRE].

[47] M. Hotta, J. Trevison and K. Yamaguchi, Gravitational memory charges of supertranslation and superrotation on Rindler horizons, Phys. Rev. D 94 (2016) 083001 [arXiv: 1606. 02443] [INSPIRE].

[48] P. Mao, X. Wu and H. Zhang, Soft hairs on isolated horizon implanted by electromagnetic fields, Class. Quant. Grav. 34 (2017) 055003 [arXiv:1606.03226] [INSPIRE].

[49] M.R. Setare and H. Adami, The Heisenberg algebra as near horizon symmetry of the black flower solutions of Chern-Simons-like theories of gravity, Nucl. Phys. B 914 (2017) 220 [arXiv: 1606.05260] [INSPIRE].

[50] D.-F. Zeng, Resolving the Schwarzschild singularity in both classic and quantum gravity, Nucl. Phys. B 917 (2017) 178 [arXiv:1606.06178] [INSPIRE].

[51] A. Averin, G. Dvali, C. Gomez and D. Lüst, Goldstone origin of black hole hair from supertranslations and criticality, Mod. Phys. Lett. A 31 (2016) 1630045 [arXiv: 1606. 06260] [INSPIRE].

[52] H. Afshar, D. Grumiller and M.M. Sheikh-Jabbari, Black hole horizon fluffs: near horizon soft hairs as microstates of three dimensional black holes, arXiv:1607.00009 [INSPIRE].

[53] M. Mirbabayi and M. Porrati, Dressed hard states and black hole soft hair, Phys. Rev. Lett. 117 (2016) 211301 [arXiv:1607.03120] [INSPIRE].

[54] D. Grumiller, A. Perez, S. Prohazka, D. Tempo and R. Troncoso, Higher spin black holes with soft hair, JHEP 10 (2016) 119 [arXiv:1607.05360] [INSPIRE]. 
[55] L. Donnay, G. Giribet, H.A. González and M. Pino, Extended symmetries at the black hole horizon, JHEP 09 (2016) 100 [arXiv:1607.05703] [INSPIRE].

[56] P. Betzios, N. Gaddam and O. Papadoulaki, The black hole S-matrix from quantum mechanics, JHEP 11 (2016) 131 [arXiv:1607.07885] [INSPIRE].

[57] J.F.M. Delgado, C.A.R. Herdeiro and E. Radu, Violations of the Kerr and Reissner-Nordström bounds: horizon versus asymptotic quantities, Phys. Rev. D 94 (2016) 024006 [arXiv: 1606.07900] [INSPIRE].

[58] M.M. Sheikh-Jabbari and H. Yavartanoo, Horizon fluffs: near horizon soft hairs as microstates of generic AdS 3 black holes, Phys. Rev. D 95 (2017) 044007 [arXiv: 1608.01293] [INSPIRE].

[59] S. Carlip, The dynamics of supertranslations and superrotations in $2+1$ dimensions, arXiv: 1608.05088 [INSPIRE].

[60] E. Gasperin and J.A.V. Kroon, Zero rest-mass fields and the Newman-Penrose constants on flat space, arXiv:1608.05716 [INSPIRE].

[61] R.-G. Cai, S.-M. Ruan and Y.-L. Zhang, Horizon supertranslation and degenerate black hole solutions, JHEP 09 (2016) 163 [arXiv:1609.01056] [INSPIRE].

[62] H.-P. Yan and W.-B. Liu, The third order correction on Hawking radiation and entropy conservation during black hole evaporation process, Phys. Lett. B 759 (2016) 293 [InSPIRE].

[63] F. Hopfmüller and L. Freidel, Gravity degrees of freedom on a null surface, arXiv: 1611.03096 [INSPIRE].

[64] R.J. Epp, The symplectic structure of general relativity in the double null $(2+2)$ formalism, gr-qc/9511060 [INSPIRE].

[65] P.R. Brady, S. Droz, W. Israel and S.M. Morsink, Covariant double null dynamics: $(2+2)$ splitting of the Einstein equations, Class. Quant. Grav. 13 (1996) 2211 [gr-qc/9510040] [INSPIRE].

[66] M. Parikh and F. Wilczek, An action for black hole membranes, Phys. Rev. D 58 (1998) 064011 [gr-qc/9712077] [INSPIRE].

[67] M.P. Reisenberger, The symplectic 2-form for gravity in terms of free null initial data, Class. Quant. Grav. 30 (2013) 155022 [arXiv:1211.3880] [INSPIRE].

[68] K. Parattu, S. Chakraborty and T. Padmanabhan, Variational principle for gravity with null and non-null boundaries: a unified boundary counter-term, Eur. Phys. J. C 76 (2016) 129 [arXiv: 1602.07546] [InSPIRE].

[69] K. Parattu, S. Chakraborty, B.R. Majhi and T. Padmanabhan, A boundary term for the gravitational action with null boundaries, Gen. Rel. Grav. 48 (2016) 94 [arXiv: 1501.01053] [INSPIRE].

[70] L. Lehner, R.C. Myers, E. Poisson and R.D. Sorkin, Gravitational action with null boundaries, Phys. Rev. D 94 (2016) 084046 [arXiv: 1609.00207] [InSPIRE].

[71] G. Hayward, Gravitational action for space-times with nonsmooth boundaries, Phys. Rev. D 47 (1993) 3275 [inSPIRE].

[72] S.W. Hawking and C.J. Hunter, The gravitational Hamiltonian in the presence of nonorthogonal boundaries, Class. Quant. Grav. 13 (1996) 2735 [gr-qc/9603050] [INSPIRE]. 
[73] Y. Neiman, Imaginary part of the gravitational action at asymptotic boundaries and horizons, Phys. Rev. D 88 (2013) 024037 [arXiv: 1305.2207] [InSPIRE].

[74] K. Schoutens, H.L. Verlinde and E.P. Verlinde, Quantum black hole evaporation, Phys. Rev. D 48 (1993) 2670 [hep-th/9304128] [INSPIRE].

[75] M. Hotta, K. Sasaki and T. Sasaki, Diffeomorphism on horizon as an asymptotic isometry of Schwarzschild black hole, Class. Quant. Grav. 18 (2001) 1823 [gr-qc/0011043] [INSPIRE].

[76] J.-I. Koga, Asymptotic symmetries on Killing horizons, Phys. Rev. D 64 (2001) 124012 [gr-qc/0107096] [INSPIRE].

[77] M. Hotta, Holographic charge excitations on horizontal boundary, Phys. Rev. D 66 (2002) 124021 [hep-th/0206222] [INSPIRE].

[78] A. Ashtekar, J. Engle, T. Pawlowski and C. Van Den Broeck, Multipole moments of isolated horizons, Class. Quant. Grav. 21 (2004) 2549 [gr-qc/0401114] [INSPIRE].

[79] W. Donnelly and A.C. Wall, Entanglement entropy of electromagnetic edge modes, Phys. Rev. Lett. 114 (2015) 111603 [arXiv:1412.1895] [INSPIRE].

[80] W. Donnelly and A.C. Wall, Geometric entropy and edge modes of the electromagnetic field, Phys. Rev. D 94 (2016) 104053 [arXiv:1506.05792] [INSPIRE].

[81] D. Harlow, Wormholes, emergent gauge fields and the weak gravity conjecture, JHEP 01 (2016) 122 [arXiv:1510.07911] [INSPIRE].

[82] J. Maldacena, D. Stanford and Z. Yang, Conformal symmetry and its breaking in two dimensional nearly anti-de-Sitter space, Prog. Theor. Exp. Phys. 2016 (2016) 12C104 [arXiv: 1606.01857] [INSPIRE].

[83] D. Harlow, The Ryu-Takayanagi formula from quantum error correction, arXiv: 1607.03901 [INSPIRE].

[84] F. Cachazo and A. Strominger, Evidence for a new soft graviton theorem, arXiv:1404.4091 [INSPIRE].

[85] G. Barnich and C. Troessaert, Symmetries of asymptotically flat 4 dimensional spacetimes at null infinity revisited, Phys. Rev. Lett. 105 (2010) 111103 [arXiv:0909.2617] [INSPIRE].

[86] G. Barnich and C. Troessaert, Supertranslations call for superrotations, PoS (CNCFG2010) 010 [Ann. U. Craiova Phys. 21 (2011) S11] [arXiv:1102.4632] [InSPIRE].

[87] G. Barnich and C. Troessaert, Aspects of the BMS/CFT correspondence, JHEP 05 (2010) 062 [arXiv: 1001.1541] [InSPIRE].

[88] G. Barnich and C. Troessaert, BMS charge algebra, JHEP 12 (2011) 105 [arXiv:1106.0213] [INSPIRE].

[89] T. Mädler and J. Winicour, Bondi-Sachs formalism, Scholarpedia 11 (2016) 33528 [arXiv: 1609.01731] [INSPIRE].

[90] P.T. Chruściel, M.A.H. MacCallum and D.B. Singleton, Gravitational waves in general relativity: 14. Bondi expansions and the polyhomogeneity of Scri, gr-qc/9305021 [INSPIRE].

[91] P.T. Chruściel and T.-T. Paetz, Characteristic initial data and smoothness of Scri I. Framework and results, Annales Henri Poincaré 16 (2015) 2131 [arXiv:1403.3558] [INSPIRE]. 
[92] D. Christodoulou and S. Klainerman, The global nonlinear stability of the Minkowski space, Princeton University Press, Princeton U.S.A., (1993) [INSPIRE].

[93] H. Bondi, M.G.J. van der Burg and A.W.K. Metzner, Gravitational waves in general relativity 7. Waves from axisymmetric isolated systems,

Proc. Roy. Soc. Lond. A 269 (1962) 21 [InSPIRE].

[94] R.K. Sachs, Gravitational waves in general relativity 8. Waves in asymptotically flat space-times, Proc. Roy. Soc. Lond. A 270 (1962) 103 [inSPIRE].

[95] S. Weinberg, Infrared photons and gravitons, Phys. Rev. 140 (1965) B516 [InSPIRE].

[96] Y. Geyer, A.E. Lipstein and L. Mason, Ambitwistor strings at null infinity and (subleading) soft limits, Class. Quant. Grav. 32 (2015) 055003 [arXiv:1406.1462] [INSPIRE].

[97] C.D. White, Diagrammatic insights into next-to-soft corrections, Phys. Lett. B 737 (2014) 216 [arXiv:1406.7184] [INSPIRE].

[98] Z. Bern, S. Davies and J. Nohle, On loop corrections to subleading soft behavior of gluons and gravitons, Phys. Rev. D 90 (2014) 085015 [arXiv: 1405.1015] [INSPIRE].

[99] F. Cachazo and E.Y. Yuan, Are soft theorems renormalized?, arXiv:1405.3413 [INSPIRE].

[100] S. He, Y.-T. Huang and C. Wen, Loop corrections to soft theorems in gauge theories and gravity, JHEP 12 (2014) 115 [arXiv: 1405.1410] [INSPIRE].

[101] Z. Bern, S. Davies, P. Di Vecchia and J. Nohle, Low-energy behavior of gluons and gravitons from gauge invariance, Phys. Rev. D 90 (2014) 084035 [arXiv:1406.6987] [InSPIRE].

[102] J. Broedel, M. de Leeuw, J. Plefka and M. Rosso, Constraining subleading soft gluon and graviton theorems, Phys. Rev. D 90 (2014) 065024 [arXiv:1406.6574] [InSPIRE].

[103] J. Broedel, M. de Leeuw, J. Plefka and M. Rosso, Local contributions to factorized soft graviton theorems at loop level, Phys. Lett. B 746 (2015) 293 [arXiv:1411.2230] [INSPIRE].

[104] D. Kapec, P. Mitra, A.-M. Raclariu and A. Strominger, A $2 D$ stress tensor for $4 D$ gravity, arXiv: 1609.00282 [INSPIRE].

[105] C. Cheung, A. de la Fuente and R. Sundrum, $4 D$ scattering amplitudes and asymptotic symmetries from $2 D$ CFT, JHEP 01 (2017) 112 [arXiv:1609.00732] [INSPIRE].

[106] T. Regge and C. Teitelboim, Role of surface integrals in the Hamiltonian formulation of general relativity, Annals Phys. 88 (1974) 286 [INSPIRE].

[107] R. Beig and N. oMurchadha, The Poincaré group as the symmetry group of canonical general relativity, Annals Phys. 174 (1987) 463 [INSPIRE].

[108] R.L. Arnowitt, S. Deser and C.W. Misner, Dynamical structure and definition of energy in general relativity, Phys. Rev. 116 (1959) 1322 [INSPIRE].

[109] R.K. Sachs, Gravitational waves in general relativity 6. The outgoing radiation condition, Proc. Roy. Soc. Lond. A 264 (1961) 309 [InSPIRE].

[110] R. Penrose, Conformal treatment of infinity, Gordon and Breach, London U.K., (1964), reprinted in Gen. Rel. Grav. 43 (2011) 901 [INSPIRE].

[111] D. Christodoulou, The global initial value problem in general relativity, in Proceedings of the Ninth Marcel Grossman Meeting on General Relativity, V. Gurzadyan, R. Jantzen and R. Ruffini eds., World Scientific, Singapore, (2002), pg. 44. 
[112] M. Dafermos, The formation of black holes in general relativity, https://www.dpmms.cam.ac.uk/ md384/expose-chr.pdf.

[113] H. Friedrich, On purely radiative space-times, Commun. Math. Phys. 103 (1986) 35 [INSPIRE].

[114] C.X. Habisohn, Massless scalar fields at null and spatial infinity in the Schwarzschild space-time, J. Math. Phys. 30 (1989) 1103 [inSPIRE].

[115] J. Corvino and R.M. Schoen, On the asymptotics for the vacuum Einstein constraint equations, J. Diff. Geom. 73 (2006) 185 [gr-qc/0301071] [InSPIRE].

[116] T.N. Palmer, Covariant conservation equations and their relation to the energy-momentum concept in general relativity, Phys. Rev. D 18 (1978) 4399 [INSPIRE].

[117] S.G. Avery and B.U.W. Schwab, Noether's second theorem and Ward identities for gauge symmetries, JHEP 02 (2016) 031 [arXiv: 1510.07038] [INSPIRE].

[118] C. Crnkovic and E. Witten, Covariant description of canonical formalism in geometrical theories, in Three hundred years of gravitation, S.W. Hawking and W. Israel eds., Cambridge University Press, Cambridge U.K., (1986), pg. 676 [INSPIRE].

[119] R.M. Wald and A. Zoupas, A general definition of 'conserved quantities' in general relativity and other theories of gravity, Phys. Rev. D 61 (2000) 084027 [gr-qc/9911095] [INSPIRE].

[120] P.-N. Chen, L.-H. Huang, M.-T. Wang and S.-T. Yau, On the validity of the definition of angular momentum in general relativity, Annales Henri Poincaré 17 (2016) 253 [arXiv: 1401.0597] [INSPIRE].

[121] A. Strominger and A. Zhiboedov, Superrotations and black hole pair creation, Class. Quant. Grav. 34 (2017) 064002 [arXiv:1610.00639] [INSPIRE].

[122] R. Penrose, The geometry of impulsive gravitational waves, in General relativity, papers in honour of J.L. Synge, L. O'Raifeartaigh ed., Clarendon Press, Oxford U.K., (1972), pg. 101 [INSPIRE].

[123] A. Ashtekar and T. Dray, On the existence of solutions to Einstein's equation with nonzero Bondi news, Commun. Math. Phys. 79 (1981) 581 [INSPIRE].

[124] M. Campiglia and A. Laddha, Sub-subleading soft gravitons: new symmetries of quantum gravity?, Phys. Lett. B 764 (2017) 218 [arXiv: 1605.09094] [INSPIRE].

[125] M. Campiglia and A. Laddha, Subleading soft photons and large gauge transformations, JHEP 11 (2016) 012 [arXiv:1605.09677] [INSPIRE].

[126] J. de Boer and S.N. Solodukhin, A holographic reduction of Minkowski space-time, Nucl. Phys. B 665 (2003) 545 [hep-th/0303006] [INSPIRE].

[127] T. Banks, A critique of pure string theory: heterodox opinions of diverse dimensions, hep-th/0306074 [INSPIRE].

[128] L. Donnay, G. Giribet, H.A. González and M. Pino, Supertranslations and superrotations at the black hole horizon, Phys. Rev. Lett. 116 (2016) 091101 [arXiv:1511.08687] [INSPIRE].

[129] C. Barrabes and W. Israel, Thin shells in general relativity and cosmology: the lightlike limit, Phys. Rev. D 43 (1991) 1129 [inSPIRE].

[130] M. Blau and M. O'Loughlin, Horizon shells and BMS-like soldering transformations, JHEP 03 (2016) 029 [arXiv: 1512.02858] [INSPIRE]. 
[131] M. Blau and M. O'Loughlin, Horizon shells: classical structure at the horizon of a black hole, Int. J. Mod. Phys. D 25 (2016) 1644010 [arXiv:1604.01181] [InSPIRE].

[132] A. Ashtekar and M. Streubel, Symplectic geometry of radiative modes and conserved quantities at null infinity, Proc. Roy. Soc. Lond. A 376 (1981) 585 [inSPIRE].

[133] G.J. Zuckerman, Action principles and global geometry, Conf. Proc. C 8607214 (1986) 259 [INSPIRE].

[134] J. Lee and R.M. Wald, Local symmetries and constraints, J. Math. Phys. 31 (1990) 725 [INSPIRE].

[135] G. Barnich and F. Brandt, Covariant theory of asymptotic symmetries, conservation laws and central charges, Nucl. Phys. B 633 (2002) 3 [hep-th/0111246] [INSPIRE].

[136] B.S. DeWitt, Quantum theory of gravity 1. The canonical theory, Phys. Rev. 160 (1967) 1113 [InSPIRE]. 